\title{
Analysis of the Behavioral, Cellular and Molecular Characteristics of Pain in Severe Rodent Spinal Cord Injury
}

Corinne A. Lee-Kubli ${ }^{1}$, Martin Ingves ${ }^{2}$, Kenneth W. Henry ${ }^{2}$, Rani Shiao ${ }^{1}$, Eileen Collyer ${ }^{1}$, Mark H. Tuszynski ${ }^{1,3,4}$, Wendy M. Campana ${ }^{2,3}$

Departments of ${ }^{1}$ Neuroscience, ${ }^{2}$ Anesthesiology, ${ }^{3}$ Program in Neurosciences, University of California, San Diego, La Jolla, CA, USA; ${ }^{4}$ Veterans Administration

Running head: Spinal Cord Injury Pain

Correspondence to: Wendy Campana, Ph.D., Department of Anesthesiology, University of California, San Diego, 9500 Gilman Drive, MTF 447, La Jolla, CA 92093-0629, USA.

Phone: 858-822-3767; Fax: 858-534-1445; Email: wcampana@ucsd.edu

Number of pages: 30

Number of figures: 9

Number of words in Abstract: 271

Number of words in Introduction: 558

Number of words in Discussion: 2035 


\section{Abstract}

Human $\mathrm{SCl}$ is frequently associated with chronic pain that is severe and refractory to medical therapy. Most rodent models used to assess pain outcomes in $\mathrm{SCl}$ apply moderate injuries to lower thoracic spinal levels, whereas the majority of human lesions are severe in degree and occur at cervical or upper thoracic levels. To better model and understand mechanisms associated with chronic pain after $\mathrm{SCl}$, we subjected adult rats to T3 severe compression or complete transection lesions, and examined pain-related behaviors for three months. Within one week after injury, rats developed consistent forepaw pain-related behaviors including increased spontaneous lifts, tactile allodynia and cold sensitivity that persisted for three months. Place escape avoidance testing confirmed that withdrawal of the forepaws from a von Frey stimulus represented active pain-related aversion. Spontaneous and evoked pain-related measures were attenuated by gabapentin, further indicating that these behaviors reflect development of pain. Spinal level of injury was relevant: rats with $\mathrm{T} 11$ severe $\mathrm{SCl}$ did not exhibit forepaw pain-related behaviors. Immunoblotting and immunofluorescence of C6-C8 spinal dorsal horn, reflecting sensory innervation of the forepaw, revealed: 1) expansion of CGRP immunoreactivity in lamina I/II; 2) increased GAP-43 expression; and 3) increased IBA1, GFAP and connexin-43 expression. These findings indicate that aberrant pain fiber sprouting and gliopathy occur after severe SCI. Notably, satellite glial cells (SGCs) in C6-C8 DRGs exhibited increases in GFAP and connexin-43, suggesting ongoing peripheral sensitization. Carbenoxolone, a gap junction inhibitor, and specific peptide inhibitors of connexin-43, ameliorated established tactile allodynia after severe $\mathrm{SCl}$. Collectively, severe T3 SCl successfully models persistent pain states and could constitute a useful model system for examining candidate translational pain therapies after SCl. 


\section{Introduction}

Pain develops in approximately two-thirds of patients with spinal cord injury (SCl) [24;54;55]. Chronic neuropathic pain is the most debilitating type of post-SCl pain, and in the majority of patients manifests as spontaneous ongoing pain with accompanying mechanical and cold allodynia [4]. Mechanisms underlying chronic post-SCI pain are poorly understood and thus, drug development for treatment has been limited and remains a high priority.

The majority of human $\mathrm{SCl}$ are severe, characterized by complete loss of sensory and motor function below the lesion, and occur most commonly at cervical or high thoracic spinal level [50]. Yet, the majority of pre-clinical models of $\mathrm{SCl}$ pain have focused on excitotoxic lesions, ischemic injuries or incomplete contusion injuries of the low thoracic spinal cord [59]. Contusion injury models are widely considered to be the most mechanistically relevant to human $\mathrm{SCl}$; however, when administered in a manner that results in injury of moderate severity, this model can spare white matter at the lesion site and allows for substantial recovery of locomotor function. In contrast, most human $\mathrm{SCl}$ is severe in degree with little, if any, recovery of neurological function. Moreover, it is difficult to assess the extent of sensory functional sparing below an incomplete rodent SCI lesion $[19,38,57]$ and to determine whether responses to sensory testing reflect pain-related behaviors rather than alterations in reflex responses, including spasticity [5]. One approach to circumvent these problems has been to evaluate symptoms of "at-level" pain in regions that are not denervated by the $\mathrm{SCl}$. Common traumatic injury models include evaluating torso allodynia after moderate thoracic contusion [34] and contralateral forelimb allodynia after cervical hemicontusion [19]. Nevertheless, the percent of rats that develop signs of allodynia in these models remains low, perhaps due to variability in tissue sparing, meaning that a much greater number of rats needs to be used to develop a mechanistic understanding of neuropathic pain following $\mathrm{SCl}$.

Although some molecular mechanisms associated with enhanced evoked withdrawal or chronic pain behaviors after SCI have been identified $[5,7,8,12,16,28$ 31,35 ] relatively little is known about mechanisms underlying chronic post-SCI pain 
behaviors in the forelimbs, particularly following injuries in the cervical or upper thoracic spinal cord [19]. Because spinal cord levels (C7/C8) mediating forelimb sensation in rats are anatomically adjacent (within $3-5 \mathrm{~mm}$ ) to cervical or upper thoracic spinal cord injuries, pain outcomes in the forelimbs likely reflect "at-level" pain, a clinically relevant type of pain experience for SCl patients [24]. Furthermore, there are few studies of "atand above-level" pain behaviors and outcomes in rodent models of the most common form of human injury, severe injury [41].

In this study, we report that forelimb pain-related behaviors consistently develop and persist following severe $\mathrm{T} 3 \mathrm{SCl}$ over a 3-month period. Forelimb pain is associated with aberrant sprouting of CGRP immunoreactive axons, but without alterations in the IB4-binding population or GAD67 immunoreactivity at C6-C8 spinal levels. Glial activation is also observed. Notably, connexin-43 and GFAP are increased in C6-C8 DRGs after severe $\mathrm{SCl}$, suggesting ongoing peripheral sensitization. Forepaw tactile allodynia is ameliorated by treatment with the gap junction decoupler, carbenoxolone, and connexin-43 peptide inhibitors, highlighting the importance of glial intercommunication in chronic pain associated with severe $\mathrm{SCl}$. Our results suggest that $\mathrm{T} 3 \mathrm{SCl}$ successfully models persistent pain states after $\mathrm{SCl}$, particularly "at-level" pain experienced by patients. Severe $\mathrm{SCl}$ constitutes a useful model system for examining effects of candidate translational pain therapies.

\section{Methods}

\section{Experimental Design}

Studies were performed using a total of 102 adult female Fischer 344 rats (150-160 g; Harlan Industries, San Diego, CA, USA). We chose female rats for these studies because their bladders can be more readily emptied following severe $\mathrm{SCl}$ compared to male rats, resulting in greater subject survival. It has been reported that pain prevalence rates and descriptions of pain do not differ between male and female patients with $\mathrm{SCl}$ $[17,62]$ and there is little evidence to suggest that female rats are less suitable for studies of pain due to the variability introduced by associated with the estrus cycle [47]. In previous studies, pain-related behaviors developed in both male and female rats after $\mathrm{SCl}$; accordingly, we used female rats. 
Experimental subjects underwent either sham surgery ( $\mathrm{N}=30), \mathrm{T} 3$ complete transection $(\mathrm{N}=30)$ or severe $\mathrm{T} 3$ compression $(\mathrm{N}=12)$. Comparisons were made to animals that underwent $\mathrm{T} 11$ transections $(\mathrm{N}=9)$. We used $\mathrm{T} 3$ level lesions because this is the highest spinal level at which a severe spinal cord lesion in the rat is consistent with subject survival; severe lesions located more rostral than T3 result in persistent forelimb dysfunction and rats are subsequently unable to move about their enclosures to access nutrition and fluid. Beginning one week after T3 severe lesions, subjects underwent biweekly assessment of spontaneous paw lifts, withdrawal thresholds using von Frey hairs and cold withdrawal threshold. Studies were performed for 12-13 weeks. Two animals were excluded at study completion; one was excluded due to an incomplete lesion (BBB score $>8$ ) and the other due to death at week 3. In additional cohorts of rats, C6-C8 DRGs and spinal dorsal horn tissues were collected 4 and 8 weeks after injury for immunoblotting and immunofluorescence ( $\mathrm{T} 3$ transected, $\mathrm{N}=13$; sham, $\mathrm{N}=11$; naïve, $\mathrm{N}=8$ ).

\section{Animal Care}

Animals were housed 2-3 per cage with free access to food and water in a vivarium approved by the American Association for the Accreditation of Laboratory Animal Care. All animal studies were carried out according to protocols approved by the Institutional Animal Care and Use Committee of the VA hospital, San Diego, CA and following the IASP Guidelines for Use of Animals in Research.

\section{Spinal cord injury and sham surgeries}

After two weeks of acclimation and collection of baseline behavioral values, rats underwent surgery under deep anesthesia using a combination of ketamine $(75 \mathrm{mg} / \mathrm{kg})$, xylazine $(2.6 \mathrm{mg} / \mathrm{ml})$, and acepromazine $(0.5 \mathrm{mg} / \mathrm{ml})$. An incision was made in the skin of the back over the T2 spinous process. After clearing of the muscle, the dorsal aspect of the T3 vertebra was removed. For severe compression, the cord was compressed for 5 seconds using $1.5 \mathrm{~mm}$-wide mosquito forceps locked and completely closed (Fig. 1). This injury procedure is a modification of the clip-compression model [47]. For 
complete transection, a longitudinal incision was made in the dura with a \#11 blade, leaving the surface of the cord exposed. Small iridectomy scissors were used to bilaterally transect the cord at two sites in the center of the T3 spinal segment located $1 \mathrm{~mm}$ apart, and the $1 \mathrm{~mm}$ segment between cuts was removed by aspiration. The muscles overlying the spinal cord were sutured and the skin incision was closed with surgical staples. Sham surgeries consisted of T3 laminectomy only. T11 complete transection was performed in the same way as T3 complete transection, only with laminectomy of the T9 vertebra, corresponding to the T11 spinal cord segment. Following surgery, rats were maintained in cages kept on heat pads $\left(37^{\circ} \mathrm{C}\right)$ for 1 week and received banamine $(1 \mathrm{mg} / \mathrm{kg})$ and ampicillin $(0.2 \mathrm{mg} / \mathrm{kg})$ in Ringer's lactate for three days. Bladder care was performed twice daily at 12 hour intervals for the first two weeks following surgery, and thereafter once daily until rats could urinate on their own, approximately four weeks after surgery. Bladders were always expressed prior to acclimation for behavioral testing. Rats were given amoxicillin their drinking water over the duration of the experiment to prevent bladder infections that could confound behavioral results.

\section{Locomotor assessment}

BBB scoring was conducted weekly beginning 1-2 weeks after the initial injury, when animals were sufficiently recovered from surgery to enable scoring. Scoring was conducted following the established protocol of BBB [6]. Movements that occurred during bladder or bowel movement were excluded. Two experienced observers conducted testing.

\section{Evaluation of pain-related behaviors}

Testing of pain-related behaviors was conducted starting 1-3 weeks following injury, to allow rats to recover from the surgical procedures. All experiments were conducted in a 
blinded fashion when possible (i.e. for drug administration studies and comparison of lesion levels) although the identities of lesioned vs. unlesioned animals were obvious.

Spontaneous forepaw lifts were determined while rats were contained in plexiglass chambers on a wire mesh grid. After 15 minutes of acclimation, spontaneous lifting of both forepaws was evaluated over a 4-minute period. This behavior frequently occurred in bursts of multiple alternating L-R paw lifts. Foot lifting associated with exploratory behavior, locomotion, body repositioning, and grooming was excluded [18].

Tactile withdrawal threshold was determined by testing rats in plexiglass chambers placed on a smooth stainless steel grid platform, allowing access from underneath the rat. The $50 \%$ paw withdrawal threshold to a series of calibrated von Frey filaments, (Kom Kare, Middletown, OH, USA) was assessed in the center of both hindpaws and forepaws before surgery (baseline) and at regular intervals following $\mathrm{SCl}$ using the up-down method [12] using a set of filaments calibrated to exert a force of $0.2-15 \mathrm{~g}$. Forepaw measurements were taken from the center of the forepaw while it was placed flat on the grid. Values from each pair of forepaws were averaged per time point.

Sensitivity to cold was measured using a Peltier device (TEC1-12706) that was attached to an aluminum heat sink and equipped with a $4 \mathrm{~mm}$ diameter aluminum rod [27]. Depending on the current, this device generates a consistent temperature ranging from $13^{\circ}-4^{\circ} \mathrm{C}$ at the tip of the rod. With rats placed on a metal grid within a plexiglass container, the cooled Peltier device was placed in contact with the center of each fore or hindpaw for 5 seconds or until a withdrawal was observed. Forepaw testing was conducted with the device cooled to $13{ }^{\circ} \mathrm{C}$ because naïve rats responded maximally at $10^{\circ} \mathrm{C}$. Testing was repeated 5 times for each paw, alternating between paws, and percent paw withdrawal was calculated for each pair of forepaws. At least 1 minute was allowed to pass before testing another paw in the same animal, and at least 5 minutes before testing the same paw. Withdrawal frequency was recorded in response to contact with the room temperature (RT) probe to control for the possibility that rats were reacting to the touch, rather than the temperature of the device. The probe was monitored periodically to verify that it was held at the expected temperature. Forepaw 
and core temperatures were collected at the conclusion of the 13-week T3 complete transection study to exclude the possibility that changes in reactivity to temperature were due to differences in thermoregulation between groups.

\section{Place-escape avoidance paradigm}

The place-escape avoidance paradigm, modified from [39], was used to evaluate active pain related aversion to forepaw stimulation with a von Frey filament 4 weeks after SCl. Animals were evaluated in a test box composed of two $(25 \times 30 \times 30)$ chambers separated by a central holding chamber $(12 \times 30 \times 30)$ with smooth stainless steel grid flooring throughout. The light chamber had clear plexiglass walls and no ceiling, while the dark chamber had opaque walls and ceiling. The animals were placed in the central holding chamber and allowed to habituate for $5 \mathrm{~min}$ after which the separating walls were removed and rats were allowed to move freely throughout the box. Upon entry into the dark chamber, rats in the von Frey (VF) group were stimulated with a $6 \mathrm{~g}$ von Frey filament, corresponding to a force one step greater than the mean $50 \%$ withdrawal threshold of the group, once every 10 seconds, alternating between forepaws. The no stimulation (NS) group received no stimulation while in the dark chamber. In both groups, no stimulation was applied once rats crossed into the light side. Each animal was tested for $20 \mathrm{~min}$, and the amount of time spent in the light side was recorded over 5 min time intervals. Time spent in the light side between 5-20 min was used as an indication of escape avoidance learning. Data were normalized to their corresponding NS group.

\section{Evaluation of pharmacological effects}

The effects of gabapentin (Tocris Bioscience, Bristol, United Kingdom) on spontaneous forepaw lifts in rats with a T3 complete transection $(\mathrm{N}=8)$ were studied over two days during the $13^{\text {th }}$ week following SCl. The effect of gabapentin on forepaw tactile withdrawal threshold was evaluated 4 weeks after $\mathrm{T} 3$ complete transection $(\mathrm{N}=6)$. Rats were randomly assigned to the saline or gabapentin groups. Pain-related behaviors were observed at baseline and for 5 hours following intraperitoneal 
administration of gabapentin (30 mg/kg i.p. dissolved in saline) or saline control. On the second day, after verifying that gabapentin effects had washed out, rats were assigned the opposite groups and the same experimental procedures were followed.

For evaluating the contribution of connexin-43 to maintenance of pain following severe $\mathrm{SCl}$, drugs or vehicle were injected in a $5 \mu \mathrm{l}$ volume, directly to the cervical spinal cord via an indwelling intrathecal (IT) catheter implanted 5 days prior to drug delivery. Catheters were constructed using an $15 \mathrm{~mm}$ long piece of polyethylene PE8 tubing (0.008 inch inner diameter, 0.014 inch outer diameter) fused with heat to a $5 \mathrm{~cm}$ long piece of PE10 tubing. A small amount of dental acrylic was used to help maintain the catheter at the base of the skull. The catheters were implanted as described elsewhere [57]. Briefly, rats were maintained under isoflurane anesthesia while the head was immobilized in a stereotaxic frame and an incision approximately $1 \mathrm{~cm}$ in length was made along the midline of the skull. A small incision was made in the underlying muscles perpendicularly to the midline of the skull, and the muscles were then blunt dissected to expose the atlanto-occipital membrane overlying the cisterna magna. A small nick was made in the membrane and the PE8 end of the catheter was gently inserted into the subarachnoid space. A small amount of saline was injected to verify that the catheter retained patency, before heat-sealing the PE10 end. Rats displaying forelimb locomotor deficits following implantation were excluded from the study. Drugs were administered in $10 \mu \mathrm{l}$ volume following by $5 \mu \mathrm{l}$ of saline to flush the catheter. The effects of carbenoxolone ( $25 \mu \mathrm{g}$, IT, dissolved in saline, Sigma-Aldrich) and glycyrrhizic acid ( $25 \mu \mathrm{g}$, IT, dissolved in saline, Sigma-Aldrich) on tactile allodynia were tested in 16 rats 4 weeks following T3 complete transection. The effects of Gap19 (13 $\mu \mathrm{g}$, IT, dissolved in dl $\mathrm{H}_{2} \mathrm{O}$, Tocris Bioscience), Gap26, Gap27 or Gap 27scramble (13 $\mu \mathrm{g}$, IT, dissolved in $\mathrm{dl} \mathrm{H}_{2} \mathrm{O}$, Anaspec) were tested in 21 rats 4 weeks following T3 complete transection. Rats were randomly assigned to treatments groups, and the experimenter remained blinded. Tactile withdrawal threshold was evaluated at baseline and over 5 hours following drug administration. Correct catheter placement was confirmed by laminectomy at the conclusion of the study. 


\section{Immunoblotting}

Rats were anesthetized with isoflurane and decapitated. C6-C8 spinal dorsal horn and DRGs were prepared in RIPA buffer (PBS with $1 \%$ triton-X100, $0.5 \%$ sodium deoxycholate, $0.1 \%$ SDS, protease inhibitor cocktail and sodium orthovanadate). The protein concentration in tissue extracts was determined by bicinchoninic acid assay. An equivalent amount of cellular protein ( $20 \mu \mathrm{g}$ per lane) was subjected to $4-20 \%$ gradient SDS-PAGE and electrotransferred to nitrocellulose membranes. The membranes were blocked with $5 \%$ nonfat dry milk in Tris- $\mathrm{HCl}$ buffered saline, $\mathrm{pH} 7.4$ with Tween 20 and incubated with the primary antibodies: GAD67 (1:5000,Millipore, MAB5406), GFAP (1:400, Chemicon, MAB360), Iba1 (1:2400, Thermo Scientific, PA5-27436) $\beta$-actin (1:10,000, Sigma-Aldrich, A2228), Tuj1 (1:1000, Covance, MMS-435P), Connexin-43 (1:400, Life Technologies, 71-0700) according to the manufacturer's recommendations. The membranes were washed and treated with horseradish peroxidase-conjugated secondary antibodies for $1 \mathrm{~h}$. Immunoblots were developed using enhanced chemiluminescence (Amersham). Blots were scanned (Cannoscan) and densitometry was performed with Image $\mathrm{J}(\mathrm{NIH})$.

\section{Immunofluorescence}

Tissue used for histology was collected from deeply anesthetized rats that were transcardially perfused with $0.9 \%$ saline followed by $4 \%$ PFA. DRGs and spinal column were removed and kept in $4 \%$ PFA overnight at $4{ }^{\circ} \mathrm{C}$ and transferred to $30 \%$ sucrose prior to dissection and sectioning. For evaluation of the spinal lesion site, $30 \mu \mathrm{M}$ horizontal tissue sections were collected every 6 sections and subsequently directly mounted onto slides and Nissl stained. Spinal cord cross-sections for IF were free floated. DRG sections were cut at $10 \mu \mathrm{M}$ thickness. Sections were incubated overnight at $4{ }^{\circ} \mathrm{C}$ with biotin-conjugated IB4 (1:1000, Sigma L2140) or primary antibodies against: CGRP (1:1000, EMD Millipore ab15360), Iba1 (1:1000; Wako Chemicals 019-19741), GFAP (for spinal cord 1:1000; EMD Millipore mab360; for DRG 1:500, Dako Z0334), NeuN (1:1000, EMD Millipore MAB377), GAD67 (1:1000, EMD Millipore MAB5406), connexin-43 (CX-43; 1:500, Everest Biotech EB09301). Appropriate Alexa-Fluor 488, 
594 or 647 secondary antibodies raised in donkey or goat (Life Technologies) were applied for $1 \mathrm{hr}$ at room temperature. In control studies, the primary antibody was omitted. Nuclei were labeled with DAPI (Invitrogen, Eugene, OR). Slides are visualized using an Olympus BX 53 microscope. Pictures were taken with a mounted camera $(Q$ Imaging Retina 2000R) and CellSens Digital Imaging computer software. Images were taken using consistent exposure times across all sections. A light level image was taken for identification of the dorsal horn. Images used for evaluation of CX-43 expression and co-localization were collected with an Olympus confocal microscope, (Fluoview FV1000-IX81) at $0.57 \mu \mathrm{M}$ intervals using a $60 \mathrm{x}$ objective lens.

Immunofluorescence was quantified by evaluating six sections sampled throughout the $\mathrm{C} 6-\mathrm{C} 8$ region using Image J software $(\mathrm{NIH})$. For evaluation of mean light intensity a region of interest was drawn around the periphery of the immunolabeled region of the dorsal horn and the fluorescent intensity within the region of interest was normalized to the mean from each naïve group. Percent area of Iba1 and GFAP was evaluated by measuring the number of pixels containing immunoreactivity above minimum threshold intensity, determined using the thresholding function of ImageJ, within the delineated dorsal horn. Connexin-43 intensity in the DRG was evaluated by taking a maximum projection Z-stack of images collected with the confocal microscope from C6, C7 and C8 DRG and calculating \% area of the image that was occupied by connexin-43 immunoreactivity. Data collected from C6-C8 spinal cord and DRG were pooled together for each experimental subject. All quantification was performed in a blinded fashion.

\section{Statistical Analysis}

Statistical analyses were performed with GraphPad Prism (v 5.0; La Jolla, CA, USA) using either unpaired one- or two-tailed t-test, paired two-tailed t-test, one-way ANOVA followed by Tukey's or Dunnett's post-hoc tests for multiple comparisons, or two-way repeated measures ANOVA followed by Bonferroni's post-hoc test, as indicated. Data are reported as group mean \pm SEM. 


\section{Results}

Functional Impairment Following T3 Transection or Compression is Severe SCI

Both T3 severe compression and T3 complete transection resulted in extensive loss of hindlimb function that was associated with a score $<3$ on the 21 point BBB locomotor scale (Fig. 1A,B). In rats with T3 severe compression, BBB scores rose slightly beginning 7 weeks following injury (Fig. 1A). Nonetheless, deficits in both groups remained severe and did not differ substantially. T3 severe compression lesion histology demonstrated a band of disrupted parenchyma across the compression site with partial surrounding cavitation (Fig. 1C). T3 complete transection injuries were characterized by a narrow lesion surrounded by inflammatory cell infiltrates (Fig. 1D). Lesion histology confirmed the absence of spared tissue in the lesion site of all rats included in the study.

Severe SCI Induces Spontaneous Forelimb Pain-Related Behaviors

Spontaneous pain is a prominent manifestation of at-level pain reported by SCI patients [4]. To determine whether spontaneous pain was present in rats that have sustained severe $\mathrm{SCl}$, we evaluated spontaneous paw lifting in the forelimbs. Forelimb measures represent responses from neural circuitry located at least two spinal segments above the lesion, and could result from plastic rearrangements evoked by the proximity to the lesion site. In control rats, spontaneous forepaw lifting behavior was negligible (fewer than $3 \mathrm{lifts} / 4 \mathrm{~min}$ ) and remained unchanged over the duration of the study (Fig. 2A,B). In contrast, rats with either T3 severe compression (Fig. 2A) or T3 complete transection (Fig. 2B) exhibited significant spontaneous forepaw lifting over 12-13 weeks compared to controls (repeated measures ANOVA, $p<0.01$, comparing T3 lesioned groups to their respective control groups). In rats with T3 severe compression, spontaneous forepaw lifting declined 8 weeks after injury ( $p<0.05$ comparing $W k 8,10$ and 12 to $W k 2$ by oneway repeated measures ANOVA followed by Tukey's test), whereas these behaviors increased over time in rats with $\mathrm{T} 3$ complete transection. The reduction in spontaneous forepaw lifting was not linked to variability of locomotor outcome as spontaneous lifting 
behavior in the three rats with BBB scores greater than 3 were not significantly different from those of the four rats with BBB scores less than 3 (by unpaired two-tailed t-test comparing average weeks 8-12 spontaneous forepaw lifts and linear regression).

T3 complete transection rats were treated with gabapentin (30 mg/kg, i.p.) following 13 weeks of injury. Gabapentin is a drug that alleviates pain in a subset of human patients with SCI [42]. Gabapentin significantly reduced spontaneous forepaw lifting behavior, with a peak efficacy 60 to $120 \mathrm{~min}$ after administration (Fig. 2C). This suggests that spontaneous forepaw lifting may be a pain-related behavior, rather than being secondary to other aspects of severe thoracic injury such as postural changes.

Next, we determined whether vertebral level of injury influenced spontaneous forepaw lifting behavior. Rats that underwent a T3 complete transection exhibited significantly increased spontaneous forepaw lifting when assessed six weeks after injury (overall ANOVA, $p<0.01$; post-hoc Tukey's $p<0.001$ comparing T3 transection to control), whereas rats that underwent T11 complete transection did not display this behavior (Fig. 2D). These findings suggest that spontaneous forepaw lifting behaviors are a manifestation of at-level pain as proximity of the injury to the cervical spinal cord is necessary for their development.

\section{Severe SCI Induces Evoked Forelimb Pain-Related Behaviors}

At-level pain sensations in human patients of $\mathrm{SCl}$ also comprise abnormal evoked responses such as allodynia in response to light touch and hypersensitivity to cold stimuli [4]. We examined both tactile and cold forelimb allodynia in rats with severe SCl. Sham control and naïve rats had a $50 \%$ forepaw withdrawal threshold that consistently remained in the non-allodynic range $(>10 \mathrm{~g}$ ) over the duration of the study (Figs. $3 A, B$ ). However, rats with $\mathrm{T} 3$ severe compression exhibited consistently reduced $50 \%$ tactile withdrawal threshold beginning 1 week post-injury and continuing through the end of the study 12 weeks later (Fig. 3A). Every rat had an average 50\% paw withdrawal value less than $6.5 \mathrm{~g}$ over the $1-12$ week period, indicating consistent development of allodynia. Rats with T3 complete transection also had consistently reduced $(<6 \mathrm{~g}) 50 \%$ 
tactile withdrawal threshold (Fig. 3B). Place escape avoidance preference (PEAP) testing confirmed that withdrawal of the forepaws from the von Frey stimulus represented active pain aversion to the stimulus. Rats with T3 complete transection spent significantly more time in the light chamber when the dark chamber was paired with stimulation by a $6 \mathrm{~g}$ von Frey filament. In contrast, sham rats spent equal time in the light chamber even when the dark chamber was paired with stimulation (Fig. $3 \mathrm{C}$ ). A single dose of gabapentin acutely alleviated established tactile allodynia for 3 hours in rats with T3 complete transection (Fig. 3D). Additionally, the proximity of the injury to the cervical spinal cord and forelimb sensory circuitry was likely related to development of tactile allodynia; reduced forepaw withdrawal threshold, indicating allodynia, was not apparent in rats after T11 complete transection (Fig. 3E).

Next, we evaluated cold allodynia in severe SCI rats. We tested the forepaws with a probe held at room temperature and at $13^{\circ} \mathrm{C}$. Sham control and naive rats maintained a consistent withdrawal frequency (approximately 50\%) over the duration of the study (Fig. 4A, C). Rats with T3 severe compression and T3 complete transection consistently exhibited increased forepaw withdrawal in response to contact with the $13^{\circ} \mathrm{C}$ cold probe, suggesting enhanced sensitivity to cold stimuli (Fig. 4A, C). Paw withdrawal frequency in response to the room temperature probe was comparable across groups at all time points (Fig.4B, D), indicating that the elevated withdrawal frequency was in response to temperature rather than contact with the probe.

To determine whether injury impaired homeostatic heat regulation, as has been reported in patients with chronic SCI [37] (this could have impacted perception of cold stimuli), core and forepaw temperature was evaluated in awake rats with T3 transection, the more severe lesion, thirteen weeks after injury. No differences were identified between rats with T3 complete transection, sham surgery or naïve groups (Fig. 4E).

Neurotransmitter and Glial Mechanisms Associated with At-Level SCI Pain

In an effort to identify cellular and molecular mechanisms that are associated with severe SCI pain-related behaviors in forelimbs, we examined known mediators of 
pain processing in the cervical spinal dorsal horn mediating forelimb sensation and movement (C7-C8). Rats with T3 transection exhibited significantly increased CGRP immunoreactivity ( $p<0.05$ comparing lesioned to naïve and sham animals; Fig. $5 A-D$ ), but not increased binding of IB4 (Fig. 5E-H) in laminae I-II of spinal dorsal gray matter 4 weeks after injury. Further, there was significantly $(p<0.05)$ more overlap between the regions innervated by CGRP and the IB4-binding population, respectively (Fig.5I-L), suggesting sprouting of pain fibers in the dorsal spinal laminae. We also observed a significant increase in the growth cone marker GAP-43 in the C6-C8 spinal dorsal horn, 8 weeks after T3 complete transection ( $p<0.05$ compared to sham control; Fig.5M). GAD67 levels were not significantly different in C6-C8 spinal dorsal horn after severe $\mathrm{SCl}$ (sham=3.02 \pm 0.22 vs. Injury=3.1 \pm 0.18 arbitrary units at 8 weeks).

Glial activation in the spinal dorsal horn is a hallmark of neuropathic pain [54] and has been associated with the development of $\mathrm{SCl}$ pain in moderate contusion injuries in rodent models [11]. Immunoreactivity of the microglial marker IBA1 was significantly increased throughout the dorsal horn in animals with $\mathrm{T} 3$ complete transection compared to naïve and sham controls (Fig. 6A-D) 4 weeks post-injury ( $p<0.05$; Fig. 6D). Immunoblotting of C6-C8 dorsal spinal cord tissue both 4 and 8 weeks after T3 complete transection further confirmed significant elevations in IBA1. Rats with $\mathrm{SCl}$ had $4-$ fold greater levels of IBA 1 compared to sham controls 4 weeks after injury $(p<0.05$, Fig. 6I) and persistent 1.6-fold elevations compared to sham animals at 8 weeks postinjury ( $p<0.05$, Fig. $6 \mathrm{~J}$ ). We also observed significantly increased GFAP immunolabeling in C6-8 spinal dorsal horn 4 weeks after complete T3 transection ( $p<0.05$ compared to naïve and sham controls; Fig. 6E-H). Immunoblotting for GFAP 4 and 8 weeks following T3 complete transection confirmed these findings: GFAP was increased 4 -fold after 4 weeks of injury ( $p<0.05$, Fig. $6 \mathrm{l}$ ), and further increased to a 6 fold difference from sham control after 8 weeks of injury $(p<0.05$, Fig. $6 \mathrm{~J})$.

The observation of increased GFAP expression led us to evaluate expression of connexin-43, the primary gap junction protein expressed by astrocytes [48,59]. Connexin-43 was significantly upregulated 4 and 8 weeks following T3 complete transection in immunoblot lysates from C6-C8 dorsal spinal cord $(p<0.05$ compared to 
sham control, Fig. 7A-C). We performed double labeling to identify the cells types within the spinal dorsal horn that express connexin-43 after injury. Connexin-43 co-localized with GFAP (Fig. 7D), confirming primary expression by astrocytes, but not NeuN (Fig. $7 \mathrm{E})$. However, connexin-43 immunoreactivity was also found in close apposition to IBA1 positive structures, suggesting that it could also be present on microglia within the dorsal horn (Fig. 7F), consistent with observations following brain stab injuries [22].

Changes in DRG Satellite Glial Cells after Severe SCI: Upregulation of GFAP and Connexin-43

Peripheral sensitization has been reported both above and below the level of injury following moderate $\mathrm{SCI}[7 ; 11 ; 58]$. GFAP levels reflect satellite glial cell (SGC) activation in the DRG that sensitize primary sensory neurons following peripheral injuryinduced neuropathic pain states [24]. We evaluated GFAP expression in the C6-C8 dorsal root ganglia (DRG) to determine whether SGCs several segments rostral to the injury site are activated after severe T3 injury. Indeed, GFAP expression was significantly upregulated in DRGs in rats 4 weeks following T3 complete transection compared to uninjured controls ( $P<0.05$; Fig. 8A).

Connexin-43 is involved in coupling between adjacent SGCs, and its upregulation following peripheral injury has been associated with the development of neuropathic pain [46]. T3 complete transection significantly upregulated connexin-43 expression in C6-C8 DRGs 4 weeks following SCl ( $\mathrm{P}<0.05$ compared to sham control; Fig. 8B). Triple labeling immunofluorescence of C7 DRGs confirmed an increase of GFAP labeling in SGCs after injury that co-localized with connexin-43 (Fig. 8C). Quantification of connexin-43 immunolabeling in the C6-C8 DRG confirmed that connexin-43 expression was increased 4 weeks after injury ( $P<0.05$ compared to naïve and sham control; Fig. 8D-E). Furthermore, the quantity of connexin-43 immunolabeling was dependent on proximity of the DRG to the lesion site, with significantly greater connexin-43 expression observed in the $\mathrm{C} 8$ and $\mathrm{C} 7 \mathrm{DRG}$ from injured rats relative to C6 (Fig. 8F). Taken together, these data indicate that molecular and cellular changes 
occur in DRG neurons rostral to the injury site following severe $\mathrm{SCl}$ that may contribute to the development of chronic pain states.

\section{Treatment with Connexin-43 Blockers Significantly Reduces Severe Post-SCI Pain}

The function of connexin-43 in pain states [14] and our observation of increased connexin-43 in both the C6-C8 spinal cord and corresponding DRGs following severe $\mathrm{SCl}$ raised the possibility that this gap junction may be operational in the maintenance of severe $\mathrm{SCl}$ pain. To test this hypothesis, rats with severe $\mathrm{SCl}$ and established pain related behaviors ( 4 weeks) were treated with carbenoxolone, which disrupts gap junctions, but is also reported to have off-target effects, including blocking voltage-gated $\mathrm{Ca}^{++}$channels, P2X7 and NMDA receptors [36] and glycyrrhizic acid, which is structurally similar to carbenoxolone and therefore shares its off-target effects, without inhibiting gap junctions [36]. Carbenoxolone ( $25 \mu \mathrm{g}, \mathrm{IT})$ significantly increased paw withdrawal threshold within 1 hour of administration, whereas glycyrrhyzic acid $(25 \mu \mathrm{g}$, IT) and saline control had no effect (Fig. 9A). Next, selective peptide blockers of connexin-43 were used [13]. Administration of Gap27 (13 $\mu \mathrm{g}$, IT), which blocks both connexin-43 and -37 [13], significantly elevated paw withdrawal threshold within 90 minutes of administration, whereas the Gap27 scramble control (Gap27-S, $13 \mu \mathrm{g}$, IT) had no effect (Fig. 9B). We also tested the effect of Gap19 (13 $\mu \mathrm{g}$, IT), which selectively blocks connexin-43 hemichannels [2], and found no effect on paw withdrawal threshold (Fig. 9B). The efficacy of Gap27 was not due to an effect on normal reflex sensitivity as it had no effect of paw withdrawal threshold in naïve and control subjects (Fig. 9C), suggesting that the efficacy in T3 transected rats is due to the pathological contribution of connexin-43 to reduced paw withdrawal threshold. Lastly, Gap26 (13 $\mu \mathrm{g}$, IT), which selectively blocks connexin-43 gap junctions and hemichannels significantly elevated paw withdrawal threshold within 30 minutes of administration relative to vehicle control (Fig. 9D). Collectively, these data suggest that blocking connexin-43-mediated communication between cells alleviates established tactile allodynia in the forepaws 4 weeks after T3 complete transection.

\section{Discussion}


Herein we demonstrate the development of consistent, significant and persistent "at-level" pain-related behaviors in two rat models of clinically relevant, severe SCI: T3 severe compression and T3 complete transection. Pain-related behaviors, including spontaneous paw lifts, tactile allodynia and cold allodynia, persisted for 3 months and are consistent with clinical reports of pain modalities that develop in patients with SCl. Motor outcome scores confirmed the ongoing severe nature of these injuries, establishing direct relevance to human injury, which is most commonly severe in extent (ASIA A)[45]. Nearly all preceding studies of pain in models of traumatic SCI have used moderate contusion models of injury that can be associated with considerable white matter sparing [11; 34]. Spared axonal projections from below the level of the lesion may contribute plastic changes that depart from mechanistic consequences that are likely after severe human injury. We propose that more clinically relevant, severe injuries merit greater exploration in translational $\mathrm{SCl}$ studies [52], including the study of $\mathrm{SCl}$ pain.

T3 complete transection and severe compression models result in major locomotor deficits and produce well-defined, consistent and clearly interpretable results [39]. As candidate regenerative therapies have now shown functional benefits in models of severe $\mathrm{SCl}$ [40], testing of pain outcomes in these severe models is becoming increasingly important for potential clinical translation. Preclinical models of $\mathrm{SCl}$ that will allow for simultaneous evaluation of functional efficacy and impact on clinically relevant neuropathic pain outcomes are needed. Furthermore, severe compression and transection lesions are consistent and reproducible such that all of the subjects in our studies exhibited pain-related behaviors across all tested modalities, in contrast to studies using moderate contusion injuries wherein only subsets of animals develop "at"or "above-level" neuropathic pain [19; 32; 33; 44].

We placed injuries in the high thoracic spinal cord (T3) and detected changes in forelimb pain-related behaviors. This likely reflects "at-level" pain due to the proximity of the lesion site to forelimb spinal cord segments that process sensory inputs (C7-C8). Cervical and high thoracic injuries constitute the majority of human injuries and are often associated with "at-level" pain [50]. Notably, forelimb pain-related behaviors 
developed in our rats with $\mathrm{T} 3$ but not $\mathrm{T} 11$ severe $\mathrm{SCl}$. While previous studies have reported that lower thoracic (T10-T13) contusion injuries can result in forelimb pain [7; $11 ; 18 ; 33]$, this may be a feature of incomplete lesion models, as models of T9 and T13 complete transection did not develop forepaw allodynia [18; 41]. Development of "above-level" pain in human patients has been reported following $\mathrm{SCl}$ [26] but is now believed to occur only in cases in which patients have co-morbid conditions, such as diabetes mellitus, that predisposes them to development of neuropathic pain [55]. The current IASP classification of SCI pain, which no longer includes "above-level" pain, defines "at-level" pain as one that is detected within three dermatomes of the injury site [48]. It is likely that pain in the forelimbs of our rats after T3 severe lesions reflects "atlevel" pain, because 3 spinal levels in humans $(\sim 30 \mathrm{~mm})$ exceed the $5-6 \mathrm{~mm}$ distance from T3 to C7-C8 spinal segments in rats. Likewise, evaluation of torso allodynia following T9 complete transection showed spread of sensitivity over a $6 \mathrm{~cm}^{2}$ region above the level of injury [41], making it reasonable that "at-level" pain following T3 complete transection would encompass the dermatomes innervating the forepaws. We chose to focus our studies on the development of "above-level" in rodents pain due to clinical relevance; "at-level" pain is persistent, disruptive to quality of life and frequently rated categorized as the most disturbing pain experienced by patients with $\mathrm{SCl}[10 ; 23$; 49].

Development of "at-level" pain in patients is frequently co-incident with or predisposes patients to development of "below-level" pain, so understanding these mechanisms may inform therapeutic options for "below-level" pain [59]. "Below-level" pain assessment in a complete injury model can be challenging to interpret because there are injury-related alterations in reflex responses to sensory stimuli; in the presence of a complete spinal cord transection, it is unlikely that these responses are consciously perceived [5]. However, in the present study we examined "at- and above" level responses to pain stimuli, wherein supraspinal nociceptive projections are not interrupted. Moreover, previous studies have revealed no alterations in "above-level" simple reflex responses and have successfully modeled "at-level" pain using ischemic, excitotoxic and contusive lesions [19;33; 56; 60]. Few studies have evaluated "at-level" pain in models of severe $\mathrm{SCl}$ [41]. Additionally, we confirmed that rats with T3 complete 
transection were capable of perceiving forelimb stimuli as being aversively painful using the PEAP, which demonstrated that injured rats spent significantly more time in the light chamber when the dark chamber was paired with intermittent stimulation (6g von Frey filament). Therefore, the responses observed in the present study appear likely to represent pain responses.

We selected our sensory modalities on the basis of clinical reports of spontaneous pain, tactile allodynia and increased cold sensitivity in patients with "atlevel" SCI pain [4]. Spontaneous pain is the most difficult to evaluate in rodents because of their obvious inability to report pain. Indeed, there are no ideal methods for measuring spontaneous pain. Thus, we evaluated ongoing spontaneous pain by evaluating forepaw lifting behavior in acclimated rats, as described by others [20]. Control rats do not spontaneously flinch their paws, but rats with both T3 complete transection and severe compression exhibited a stereotypic lifting behavior that was not associated with postural adjustment. This behavior may be comparable to spontaneous foot lifting behaviors after injury which correlate with spontaneous firing of primary afferents [20]. Furthermore, forepaw lifting was alleviated by gabapentin, a drug that partially alleviates neuropathic pain in humans [42] and rodents [8], suggesting that forepaw lifting behavior was indicative of spontaneous pain. However, gabapentin has also been reported to have effects on sedation [25] and spasticity [38], and could have reduced the number of spontaneous forepaw lifts by impacting these systems. Yet, development of spasticity is typically reported only in deafferented regions below the level of the injury [3], and the dose of gabapentin we used $(30 \mathrm{mg} / \mathrm{kg})$ is below the dose reported to yield sedation [25; 31].

Rats with severe $\mathrm{T} 3 \mathrm{SCl}$ also consistently developed forelimb tactile allodynia, which was reversed by gabapentin, and increased sensitivity to cold, both of which have been reported by patients with "at-level" pain [26]. "At-level" cold allodynia has previously been reported in a model of photochemically-induced ischemic injury [27], while conscious aversion to cold flooring has been reported in a rat model of spinal stenosis [53]. Acute "at-level" cold sensitivity to acetone has also been reported following T9 complete transection [41]. Here, we have shown that, not only does cold 
allodynia manifest in the forepaws of rats with T3 severe lesions, it persists over at least a 12-13 week duration following injury. Taken together, our results suggest that severe T3 injury equally recapitulate signs of neuropathic pain that are experienced by humans who have sustained $\mathrm{SCl}$, reflecting the relevance of the severe $\mathrm{T} 3$ lesion to the clinical condition.

The altered responses of both neurons and glia play key roles in ongoing neuropathic pain [20;54]. Severe SCI induced an expansion of peptidergic axons (CGRP) in lamina I/II. No changes were observed in non-peptidergic afferents (IB4binding population). Sprouting of new peptidergic branches into $\mathrm{C} 6-\mathrm{C} 8$ spinal dorsal horn was confirmed by expression of GAP43, a growth cone marker. These findings are similar to those reported after moderate injuries, reflecting expanded nociceptive inputs to dorsal horn neurons $[17 ; 20 ; 54]$. However, in contrast to $\mathrm{SCl}$ of moderate severity, we did not observe changes in spinal GABAergic tone (altered GAD67 expression), which is thought to be associated with a greater risk of developing neuropathic pain $[21 ; 29 ; 43]$. Thus, severe $\mathrm{SCl}$ is associated with mechanisms of neuronal plasticity that are both common and distinct from previous reports in models of incomplete injury. Increased expression of excitatory neurotransmission has been reported in SCI [15; 19].

Glial cells are important for maintaining neuro-architecture and physiological homeostasis in the spinal cord [54]. After SCl, spinal glia become activated, a feature that is considered to be one of the mechanistic underpinnings of neuropathic pain [29]. After severe T3 SCI, IBA1 and GFAP expression are significantly increased in cervical spinal segments; interestingly, IBA1 is increased to a greater extent at 4 weeks than 8 weeks. This may be due to rapid mobilization of microglia and macrophages that may limit the severity of parenchymal injury, although specific macrophage responses can also worsen injury [28]. In contrast, GFAP expression was significantly increased to a greater extent at 8 weeks after injury, as compared to 4 weeks. Our findings are consistent with models of neuropathic, inflammatory and post-operative pain, which report an increase in the number of activated microglia within a few days, followed by astrocyte activation. As microglial activation begins to attenuate, astrocyte activation 
and hypersensitivity persists [30; 50; 51]. Chronic pain may be maintained, rather than initiated, by astrocyte activation, which has important implications for the development of potential therapeutics for $\mathrm{SCl}$ pain. Furthermore, targeting spinal astrocytes several segments rostral to the injury site may be beneficial in $\mathrm{SCl}$.

We describe novel cellular responses in the DRG innervating the lower cervical segments, including increased expression of GFAP and connexin-43 in SGCs, following development of forepaw pain-related behaviors. Thus, severe $\mathrm{SCl}$ involves alterations in sensory processing pathways in the pain circuit that are located outside the spinal cord. Indeed, moderate contusion injuries lead to peripheral sensitization that can be linked to alterations in intrinsic properties of primary neurons $[7 ; 11 ; 51 ; 58]$. In this study, we identified a novel extrinsic mechanism within the DRG that may regulate peripheral sensitization: ongoing activation of SGCs and upregulation of connexin-43. Connexin-43 is a gap junction protein that is involved in ion transport and communication between adjacent SGCs [46] and can also contribute to release of neurotransmitters and cytokines through hemichannels that open into the extracellular space [9]. The contribution of connexin-43 in peripheral glial activation in DRGs has not been previously appreciated in the context of persistent SCI pain. However, increased connexin-43 expression in SGCs has been shown to contribute to the development of neuropathic pain states following peripheral nerve injury [35].

A potential role for connexin-43 in severe $\mathrm{SCl}$ pain is supported by our data demonstrating that IT administration of the gap junction blocker carbenoxolone directly to the C7-C8 spinal segments reduced tactile allodynia 4 weeks following $\mathrm{SCl}$. Furthermore, IT delivery of Gap27, which blocks connexin-43 and -37, and Gap26, which selectively blocks connexin-43, robustly alleviated forepaw tactile allodynia as well. In contrast, Gap19, which selectively blocks connexin-43 hemichannels through which neurotransmitters could be released, had no effect, suggesting that elevated connexin-43 maintains pain-like behaviors primarily through enhanced coupling between adjacent cells. These drugs had no effect on forepaw sensitivity in naïve and sham rats. Though the primary site of drug delivery through an IT catheter is the spinal cord itself, intrathecally delivered agents also have access to the DRG [1], making it 
likely that drug delivery would have targeted gap junctions in both the DRG and spinal cord. Others have shown that mice with genetic deletion of connexin-43 in astrocytes had attenuated hindlimb withdrawal to tactile and heat stimuli following moderate T10 $\mathrm{SCI}$ [14], although it should be noted that cre expression in this experiment was under the GFAP promoter and could also have impacted connexin-43 levels in peripheral SGCs. Therefore, the relative contribution of SGCs in the periphery or astrocytes in the spinal cord to ongoing severe $\mathrm{SCl}$ pain related behaviors requires further study. Nonetheless, connexin-43 blockade or downregulation may be a viable therapeutic target for both treatment of neuropathic pain and improving regeneration following $\mathrm{SCl}$, as treatment with an anti-sense oligonucleotide against connexin-43 reduced tissue swelling and disruption and improved locomotor outcomes in a model of moderate compressive SCI [16].

We describe a model of $\mathrm{T} 3$ severe $\mathrm{SCl}$ that consistently results in forelimb painrelated behaviors that are comparable to reports of pain experienced by patients with $\mathrm{SCl}$. Gliopathy is associated with the development and maintenance of the chronic pain state, including peripheral alterations in connexin- 43 and central alterations in astrocytes, microglia and neurotransmitters. Notably, therapeutic targeting of the glialneuronal interface significantly ameliorated SCl-related pain.

Acknowledgements: This work was supported by the NIH (R01 NS-057456, NS042291, EB014986), the California Institute for Regenerative Medicine,Veterans Administration and the Department of Defense (SC140273). The authors declare no competing financial interests. 


\section{References}

[1] Abram SE, Yi J, Fuchs A, Hogan QH. Permeability of injured and intact peripheral nerves and dorsal root ganglia. Anesthesiology 2006;105(1):146-153.

[2] Abudara V, Bechberger J, Freitas-Andrade M, De Bock M, Wang N, Bultynck G, Naus CC, Leybaert L, Giaume $C$. The connexin43 mimetic peptide Gap19 inhibits hemichannels without altering gap junctional communication in astrocytes. Front Cell Neurosci 2014;8:306.

[3] Adams MM, Hicks AL. Spasticity after spinal cord injury. Spinal Cord 2005;43(10):577-586.

[4] Baastrup C, Finnerup NB. Pain in spinal cord injury. Pain Manag 2012;2(1):87-94.

[5] Baastrup C, Maersk-Moller CC, Nyengaard JR, Jensen TS, Finnerup NB. Spinal-, brainstem- and cerebrally mediated responses at- and below-level of a spinal cord contusion in rats: evaluation of pain-like behavior. Pain 2010;151(3):670-679.

[6] Basso DM, Beattie MS, Bresnahan JC. A sensitive and reliable locomotor rating scale for open field testing in rats. J Neurotrauma 1995;12(1):1-21.

[7] Bedi SS, Yang Q, Crook RJ, Du J, Wu Z, Fishman HM, Grill RJ, Carlton SM, Walters ET. Chronic spontaneous activity generated in the somata of primary nociceptors is associated with painrelated behavior after spinal cord injury. J Neurosci 2010;30(44):14870-14882.

[8] Bennett MI, Simpson KH. Gabapentin in the treatment of neuropathic pain. Palliat Med 2004;18(1):511.

[9] Bennett MV, Contreras JE, Bukauskas FF, Saez JC. New roles for astrocytes: gap junction hemichannels have something to communicate. Trends Neurosci 2003;26(11):610-617.

[10] Cardenas DD, Turner JA, Warms CA, Marshall HM. Classification of chronic pain associated with spinal cord injuries. Arch Phys Med Rehabil 2002;83(12):1708-1714.

[11] Carlton SM, Du J, Tan HY, Nesic O, Hargett GL, Bopp AC, Yamani A, Lin Q, Willis WD, Hulsebosch CE. Peripheral and central sensitization in remote spinal cord regions contribute to central neuropathic pain after spinal cord injury. Pain 2009;147(1-3):265-276.

[12] Chaplan SR, Bach FW, Pogrel JW, Chung JM, Yaksh TL. Quantitative assessment of tactile allodynia in the rat paw. J Neurosci Methods 1994;53(1):55-63.

[13] Chen G, Park CK, Xie RG, Berta T, Nedergaard M, Ji RR. Connexin-43 induces chemokine release from spinal cord astrocytes to maintain late-phase neuropathic pain in mice. Brain 2014;137(Pt 8):2193-2209.

[14] Chen MJ, Kress B, Han X, Moll K, Peng W, Ji RR, Nedergaard M. Astrocytic CX43 hemichannels and gap junctions play a crucial role in development of chronic neuropathic pain following spinal cord injury. Glia 2012;60(11):1660-1670.

[15] Christensen MD, Hulsebosch CE. Spinal cord injury and anti-NGF treatment results in changes in CGRP density and distribution in the dorsal horn in the rat. Exp Neurol 1997;147(2):463-475.

[16] Cronin M, Anderson PN, Cook JE, Green CR, Becker DL. Blocking connexin43 expression reduces inflammation and improves functional recovery after spinal cord injury. Mol Cell Neurosci 2008;39(2):152-160.

[17] Darian-Smith C. Primary afferent terminal sprouting after a cervical dorsal rootlet section in the macaque monkey. J Comp Neurol 2004;470(2):134-150.

[18] Densmore VS, Kalous A, Keast JR, Osborne PB. Above-level mechanical hyperalgesia in rats develops after incomplete spinal cord injury but not after cord transection, and is reversed by amitriptyline, morphine and gabapentin. Pain 2010;151(1):184-193.

[19] Detloff MR, Smith EJ, Quiros Molina D, Ganzer PD, Houle JD. Acute exercise prevents the development of neuropathic pain and the sprouting of non-peptidergic (GDNF- and arteminresponsive) c-fibers after spinal cord injury. Exp Neurol 2014;255:38-48. 
[20] Djouhri L, Koutsikou S, Fang X, McMullan S, Lawson SN. Spontaneous pain, both neuropathic and inflammatory, is related to frequency of spontaneous firing in intact C-fiber nociceptors. J Neurosci 2006;26(4):1281-1292.

[21] Eaton MJ, Plunkett JA, Karmally S, Martinez MA, Montanez K. Changes in GAD- and GABAimmunoreactivity in the spinal dorsal horn after peripheral nerve injury and promotion of recovery by lumbar transplant of immortalized serotonergic precursors. J Chem Neuroanat 1998;16(1):57-72.

[22] Eugenin EA, Eckardt D, Theis M, Willecke K, Bennett MV, Saez JC. Microglia at brain stab wounds express connexin 43 and in vitro form functional gap junctions after treatment with interferongamma and tumor necrosis factor-alpha. Proc Natl Acad Sci U S A 2001;98(7):4190-4195.

[23] Felix ER, Cruz-Almeida Y, Widerstrom-Noga EG. Chronic pain after spinal cord injury: what characteristics make some pains more disturbing than others? J Rehabil Res Dev 2007;44(5):703715.

[24] Fenzi F, Benedetti MD, Moretto G, Rizzuto N. Glial cell and macrophage reactions in rat spinal ganglion after peripheral nerve lesions: an immunocytochemical and morphometric study. Arch Ital Biol 2001;139(4):357-365.

[25] Field MJ, Oles RJ, Lewis AS, McCleary S, Hughes J, Singh L. Gabapentin (neurontin) and S-(+)-3isobutylgaba represent a novel class of selective antihyperalgesic agents. $\mathrm{Br} \mathrm{J}$ Pharmacol 1997;121(8):1513-1522.

[26] Finnerup NB, Johannesen IL, Fuglsang-Frederiksen A, Bach FW, Jensen TS. Sensory function in spinal cord injury patients with and without central pain. Brain 2003;126(Pt 1):57-70.

[27] Gao T, Hao JX, Wiesenfeld-Hallin Z, Xu XJ. Quantitative test of responses to thermal stimulation in spinally injured rats using a Peltier thermode: a new approach to study cold allodynia. J Neurosci Methods 2013;212(2):317-321.

[28] Gaudet AD, Popovich PG, Ramer MS. Wallerian degeneration: gaining perspective on inflammatory events after peripheral nerve injury. J Neuroinflammation 2011;8:110.

[29] Gwak YS, Crown ED, Unabia GC, Hulsebosch CE. Propentofylline attenuates allodynia, glial activation and modulates GABAergic tone after spinal cord injury in the rat. Pain 2008;138(2):410-422.

[30] Hald A, Nedergaard S, Hansen RR, Ding M, Heegaard AM. Differential activation of spinal cord glial cells in murine models of neuropathic and cancer pain. Eur J Pain 2009;13(2):138-145.

[31] Hao JX, XU XJ, Urban L, Wiesenfeld-Hallin Z. Repeated administration of systemic gabapentin alleviates allodynia-like behaviors in spinally injured rats. Neurosci Lett 2000;280(3):211-214.

[32] Hubscher $\mathrm{CH}$, Johnson RD. Chronic spinal cord injury induced changes in the responses of thalamic neurons. Exp Neurol 2006;197(1):177-188.

[33] Hulsebosch CE, Hains BC, Crown ED, Carlton SM. Mechanisms of chronic central neuropathic pain after spinal cord injury. Brain Res Rev 2009;60(1):202-213.

[34] Hulsebosch CE, Xu GY, Perez-Polo JR, Westlund KN, Taylor CP, McAdoo DJ. Rodent model of chronic central pain after spinal cord contusion injury and effects of gabapentin. J Neurotrauma 2000;17(12):1205-1217.

[35] Jasmin L, Vit JP, Bhargava A, Ohara PT. Can satellite glial cells be therapeutic targets for pain control? Neuron Glia Biol 2010;6(1):63-71.

[36] Juszczak GR, Swiergiel AH. Properties of gap junction blockers and their behavioural, cognitive and electrophysiological effects: animal and human studies. Prog Neuropsychopharmacol Biol Psychiatry 2009;33(2):181-198.

[37] Khan S, Plummer M, Martinez-Arizala A, Banovac K. Hypothermia in patients with chronic spinal cord injury. J Spinal Cord Med 2007;30(1):27-30.

[38] Kitzman PH, Uhl TL, Dwyer MK. Gabapentin suppresses spasticity in the spinal cord-injured rat. Neuroscience 2007;149(4):813-821. 
[39] LaBuda CJ, Fuchs PN. A behavioral test paradigm to measure the aversive quality of inflammatory and neuropathic pain in rats. Exp Neurol 2000;163(2):490-494.

[40] Lu P, Wang Y, Graham L, McHale K, Gao M, Wu D, Brock J, Blesch A, Rosenzweig ES, Havton LA, Zheng B, Conner JM, Marsala M, Tuszynski MH. Long-distance growth and connectivity of neural stem cells after severe spinal cord injury. Cell 2012;150(6):1264-1273.

[41] M'Dahoma S, Bourgoin S, Kayser V, Barthelemy S, Chevarin C, Chali F, Orsal D, Hamon M. Spinal cord transection-induced allodynia in rats--behavioral, physiopathological and pharmacological characterization. PLoS One 2014;9(7):e102027.

[42] Mehta S, McIntyre A, Dijkers M, Loh E, Teasell RW. Gabapentinoids Are Effective in Decreasing Neuropathic Pain and Other Secondary Outcomes after Spinal Cord Injury: A Meta-Analysis. Arch Phys Med Rehabil 2014.

[43] Meisner JG, Marsh AD, Marsh DR. Loss of GABAergic interneurons in laminae I-III of the spinal cord dorsal horn contributes to reduced GABAergic tone and neuropathic pain after spinal cord injury. J Neurotrauma 2010;27(4):729-737.

[44] Mills CD, Hains BC, Johnson KM, Hulsebosch CE. Strain and model differences in behavioral outcomes after spinal cord injury in rat. J Neurotrauma 2001;18(8):743-756.

[45] National Spinal Cord Injury Statistical Center Birmingham AUoAaB. 2011 Annual Statistical Report for the Spinal Cord Injury Model Systems. 2011.

[46] Ohara PT, Vit JP, Bhargava A, Jasmin L. Evidence for a role of connexin 43 in trigeminal pain using RNA interference in vivo. J Neurophysiol 2008;100(6):3064-3073.

[47] Poon PC, Gupta D, Shoichet MS, Tator CH. Clip compression model is useful for thoracic spinal cord injuries: histologic and functional correlates. Spine (Phila Pa 1976) 2007;32(25):2853-2859.

[48] Siddall PJ, Finnerup NB. Chapter 46 Pain following spinal cord injury. Handb Clin Neurol 2006;81:689-703.

[49] Siddall PJ, McClelland JM, Rutkowski SB, Cousins MJ. A longitudinal study of the prevalence and characteristics of pain in the first 5 years following spinal cord injury. Pain 2003;103(3):249-257.

[50] Svensson $\mathrm{Cl}$, Brodin E. Spinal astrocytes in pain processing: non-neuronal cells as therapeutic targets. Mol Interv 2010;10(1):25-38.

[51] Tang XQ, Tanelian DL, Smith GM. Semaphorin3A inhibits nerve growth factor-induced sprouting of nociceptive afferents in adult rat spinal cord. J Neurosci 2004;24(4):819-827.

[52] Tuszynski MH, Steward O. Concepts and methods for the study of axonal regeneration in the CNS. Neuron 2012;74(5):777-791.

[53] Vierck CJ, Baastrup C, Maersk-Moller C, Roth M, Cannon RL, Finnerup NB, Yezierski RP. A preclinical model of hyperalgesia following spinal stenosis/compression. Eur J Pain 2015.

[54] Watkins LR, Wieseler-Frank J, Milligan ED, Johnston I, Maier SF. Chapter 22 Contribution of glia to pain processing in health and disease. Handb Clin Neurol 2006;81:309-323.

[55] Widerstrom-Noga E, Biering-Sorensen F, Bryce T, Cardenas DD, Finnerup NB, Jensen MP, Richards JS, Siddall PJ. The international spinal cord injury pain basic data set. Spinal Cord 2008;46(12):818-823.

[56] Xu XJ, Hao JX, Aldskogius H, Seiger A, Wiesenfeld-Hallin Z. Chronic pain-related syndrome in rats after ischemic spinal cord lesion: a possible animal model for pain in patients with spinal cord injury. Pain 1992;48(2):279-290.

[57] Yaksh TL, Rudy TA. Chronic catheterization of the spinal subarachnoid space. Physiol Behav 1976;17(6):1031-1036.

[58] Yang Q, Wu Z, Hadden JK, Odem MA, Zuo Y, Crook RJ, Frost JA, Walters ET. Persistent pain after spinal cord injury is maintained by primary afferent activity. J Neurosci 2014;34(32):1076510769. 
[59] Yezierski RP. Spinal cord injury: a model of central neuropathic pain. Neurosignals 2005;14(4):182193.

[60] Yezierski RP, Liu S, Ruenes GL, Kajander KJ, Brewer KL. Excitotoxic spinal cord injury: behavioral and morphological characteristics of a central pain model. Pain 1998;75(1):141-155. 
Figure 1. Comparison of BBB scores in models of severe SCl. A. T3 severe compression $(n=7)$. B. T3 complete transection $(n=8)$. Data are expressed as mean \pm SEM. C. Terminal lesion histology shows a band of disrupted parenchyma with associated cavitation in rats with T3 severe compression. $\boldsymbol{D}$. Rats with T3 compression showed a narrow lesion surrounded by inflammatory cell infiltrates. Horizontal sections through the lesion site are oriented rostral to caudal from left to right. Scale bar is $1 \mathrm{~mm}$.

Figure 2. $\mathrm{T} 3$ severe $\mathrm{SCl}$ induces forepaw spontaneous pain-related behaviors in rats with $\boldsymbol{A}$. T3 severe compression compared to sham control. $\boldsymbol{B}$. T3 complete transection compared to naïve and sham control. Graphs show the number of forepaw lifts within 4 minutes over 13 weeks. $\mathrm{N}=6$-9/group. ${ }^{*} \mathrm{p}<0.05,{ }^{* *} \mathrm{p}<0.01,{ }^{* * *} \mathrm{p}<0.001$ compared to sham control and naive by two-way repeated measures ANOVA with Bonferroni post-hoc analysis. $\boldsymbol{C}$. Effect of gabapentin (30 $\mathrm{mg} / \mathrm{kg}$ i.p.) on spontaneous forepaw lifts when administered at 13 weeks after T3 complete transection. $N=8$ /group. ${ }^{* *} p<0.01$, ${ }^{* * *} p<0.001$ compared to saline treatment by two-way repeated measures ANOVA followed by Bonferroni post-hoc analysis. $D$. Effect of spinal level of injury on spontaneous forepaw lifts 6 weeks after injury in sham control , T3 complete transection (T3 Trans) and T11 complete transection (T11 Trans). $\mathrm{N}=4$ /group. ${ }^{* *} \mathrm{p}<0.01,{ }^{* * *} \mathrm{p}<0.001$ by one-way ANOVA followed by Tukey's post-hoc test. Data are expressed as mean \pm SEM.

Figure 3. Forepaw tactile allodynia develops after severe SCI. A. T3 severe compression compared to sham control. B. T3 complete transection compared to naïve and sham control. Forepaw tactile withdrawal thresholds were evaluated in rats over 13 weeks. $\mathrm{N}=6-9$ /group. ${ }^{*} \mathrm{p}<0.05,{ }^{* * *} \mathrm{p}<0.001$ compared to sham control and naive by two-way repeated measures ANOVA followed by Bonferroni post-hoc analysis. C. Place escape avoidance paradigm (PEAP) conducted 4 weeks after sham or T3 complete transection surgery. Data are expressed as "fold-difference" compared to non-stimulated (NS) $\mathrm{N}=5$ /group. ${ }^{*} \mathrm{p}<0.05$ compared to NS by unpaired, two-tailed t-test. ${ }^{* * *} p<0.001$ compared to sham by unpaired, two-tailed t-test $\boldsymbol{D}$. Effect of gabapentin (30 
$\mathrm{mg} / \mathrm{kg}$ i.p.) on forepaw tactile withdrawal when administered at 4 weeks after T3 complete transection ( $n=6 /$ group). ${ }^{* * *} p<0.001$ compared to saline treatment by two-way repeated measures ANOVA followed by Bonferroni post-hoc analysis. E. Effect of spinal level of injury on tactile withdrawal 10 weeks after injury in T3 complete transection (T3 Trans) and T11 complete transection (T11 Trans). N=4/group. ${ }^{* *} p<0.01,{ }^{* * *} p<0.01$ by one-way ANOVA followed by Tukey's post-hoc test. Data are expressed as mean \pm SEM.

Figure 4. Cold allodynia develops in the forepaws after T3 severe $\mathrm{SCl}$. A. T3 severe compression in response to the probe held at $13^{\circ} \mathrm{C}$, compared to sham control. B. T3 severe compression in response to the probe held at room temperature (RT). C. T3 complete transection in response to to the probe held at $13^{\circ} \mathrm{C}$, compared to naïve and sham control. D. T3 complete transection in response to the probe held at RT. Sensitivity to cold was assessed by withdrawal frequency in response to contact with at $13^{\circ} \mathrm{C}$ Peltier probe. $\mathrm{N}=6$-9/group. ${ }^{* *} \mathrm{p}<0.01,{ }^{* * *} \mathrm{p}<0.001$ compared to sham control and naive by two-way repeated measures ANOVA followed by Bonferroni post-hoc analysis. $E$. Core and forepaw surface temperature in naïve, sham control and rats with T3 complete transection 13 weeks following injury. $\mathrm{N}=8-9 /$ group. No significant difference between groups by one way ANOVA. Data are expressed as mean \pm SEM.

Figure 5. Alterations in CGRP expression and intensity of the IB4-binding population in C6-C8 spinal dorsal horn after severe SCl. Immunofluorescence microscopy was performed to detect CGRP in $\boldsymbol{A}$. Naïve $\boldsymbol{B}$. Sham control and $\boldsymbol{C}$. T3 complete transected rats. $D$. Pooled quantification of C6-C8 CGRP immunofluorescence intensity. IB4binding in $\boldsymbol{E}$. Naïve $\boldsymbol{F}$. Sham control and $\boldsymbol{G}$. T3 complete transected rats. $\boldsymbol{H}$. Pooled quantification of C6-C8 IB4 immunofluorescence intensity. Area of CGRP+ afferents and IB4-binding overlap in $\boldsymbol{I}$. Naïve $\boldsymbol{J}$. Sham control and $\boldsymbol{K}$. T3 complete transected rats. L. Pooled quantification of C6-C8 CGRP immunofluorescence and IB4-binding intensity. Quantification of immunofluorescence intensity is expressed as mean $\pm \mathrm{SEM}$. N=3$5 /$ group. * $p<0.01$ compared to naïve sham control by one-way ANOVA. Scale bar $=200$ 
$\mu \mathrm{M}$. M. GAP-43 levels were determined by immunoblot analysis in extracts of rat C6-C8 cervical dorsal horn 4 weeks after T3 complete transection. Blots were re-probed for $\beta$ actin as a loading control. Two representative rats from each group are shown. Equal amounts of cellular protein $(20 \mu \mathrm{g})$ were loaded into each lane and subjected to SDSPAGE. $N=4 /$ group. ${ }^{*} \mathrm{p}<0.05$ by t-test compared to sham control. Results are expressed as mean $\pm \mathrm{SEM}$.

Figure 6. Increased IBA1 and GFAP expression in C6-C8 spinal dorsal horn after severe SCl. Immunofluorescence microscopy was performed to detect IBA1 in $\boldsymbol{A}$. Naïve $\boldsymbol{B}$. Sham control and $\boldsymbol{C}$. T3 complete transected rats 4 weeks after injury. $\boldsymbol{D}$. Pooled quantification of C6-C8 IBA1 immunofluorescence intensity. GFAP expression in $\boldsymbol{E}$. Naïve $\boldsymbol{F}$. Sham control and $\boldsymbol{G}$. T3 complete transected rats 4 weeks after injury. $\boldsymbol{H}$. Pooled quantification of C6-C8 CGRP immunofluorescence intensity. Quantification of immunofluorescence intensity is expressed as mean \pm SEM. $n=3-5 /$ group. ${ }^{*} p<0.01$ compared to naïve sham control by one-way ANOVA. Scale bar $=200 \mu \mathrm{M}$. Iba1 and GFAP levels were determined by immunoblot analysis in extracts of rat C6-C8 cervical dorsal spinal cord $\boldsymbol{I} .4$ weeks and $\boldsymbol{J}$. 8 weeks after T3 complete transection. Blots were re-probed for $\beta$-actin as a loading control. Two representative rats from each group are shown. Equal amounts of cellular protein $(20 \mu \mathrm{g})$ were loaded into each lane and subjected to SDS-PAGE. N=4/group. ${ }^{*} p<0.05,{ }^{* *} p<0.01$ by t-test compared to sham control. Data are expressed as mean \pm SEM.

Figure 7. Increased connexin-43 expression in C6-C8 spinal dorsal horn following T3 complete transection. Connexin-43 at $\boldsymbol{A} .4$ weeks after $\mathrm{SCl}$ and $\boldsymbol{B} .8$ weeks after $\mathrm{SCl}$ Connexin-43 were determined by immunoblot anlaysis. Blots were re-probed for $\beta$ neuronal tubulin (Tuj1) as a loading control. Two representative rats from each group are shown. Equal amounts of cellular protein $(20 \mu \mathrm{g})$ were loaded into each lane and subjected to SDS-PAGE. C. Quantification of immunoblot results is expressed as mean \pm SEM. $N=4$ /group. ${ }^{*} p<0.05,{ }^{* *} p<0.01$ by t-test compared to sham control. Dual labeling immunofluorescence microscopy was performed to detect $\boldsymbol{D}$. connexin-43 (CX-43) and GFAP, E. CX-43 and NeuN, and F. CX-43 and IBA1 in T3 complete transected rats 4 
weeks after injury. Z-stack images generated with $0.57 \mu \mathrm{M}$ optical sections. Scale bar $=$ $10 \mu \mathrm{M}$.

Figure 8. SGCs become active in DRGs several segments rostral to injury site. $\boldsymbol{A}$. GFAP levels and $\boldsymbol{B}$. Connexin-43 were determined by immunoblot analysis in extracts of rat C6-C8 DRGs 4 weeks after injury. Blots were re-probed for $\beta$-neuronal tubulin (Tuji) as a loading control. Two representative rat DRGs are shown. Equal amounts of cellular protein $(10 \mu \mathrm{g})$ were loaded into each lane and subjected to SDS-PAGE. Quantification of immunoblot results is expressed as mean \pm SEM. N=3-9/group. ${ }^{*} p<0.05,{ }^{* *} p<0.01$ by $t$-test compared to sham control. Dual labeling immunofluorescence microscopy was performed to detect $C$. Connexin-43 (green), GFAP (red) and NeuN (cyan) expression in a C7 DRG from a sham and T3 transected rat, 4 weeks after injury. Connexin-43 is primarily expressed in the perineuronal region surrounding NeuN, and co-localizes with GFAP following complete transection. $\boldsymbol{D}$. Maximum projection z-stack of triple labeling immunofluorescence including connexin43, GFAP and NeuN expression in the C7 DRG collected from naïve, sham and T3 complete transected rats. E. Pooled quantification of the percent area of connexin-43 immunoreactivity in C6-C8 DRG. F. Quantification of the percent area of connexin-43 immunoreactivity separated into C6-C8 DRGs collected from rats 4 weeks following T3 complete transection. Data are expressed as mean \pm SEM. $n=3-5 /$ group. ${ }^{*} p<0.05$, ${ }^{* *} p<0.01$ compared to C6 by one-way ANOVA followed by Tukey's post-hoc test.

Figure 9. Blocking connexin-43 alleviates established allodynia after T3 complete transection $\boldsymbol{A}$. Effects of carbenoxolone ( $25 \mu \mathrm{g} \mathrm{IT})$ or glycyrrhyzic acid $(25 \mu \mathrm{g} \mathrm{IT})$ on tactile withdrawal threshold 4 weeks after T3 complete transection. B. Effects of Gap27scramble, Gap27 or Gap19 (13 $\mathrm{gg}$ IT) on forepaw tactile withdrawal threshold 4 weeks after T3 complete transection. $C$. Effects of Gap27 (13 $\mu \mathrm{g} \mathrm{IT)} \mathrm{on} \mathrm{forepaw} \mathrm{withdrawal}$ threshold in naïve or sham control rats. $\boldsymbol{D}$. Effects of Gap26 (13 $\mu \mathrm{g}$ IT) or vehicle control on forepaw withdrawal threshold 4 weeks after complete transection. Forepaw tactile withdrawal threshold is expressed as mean \pm SEM. $N=6-7 /$ treatment group. 
${ }^{*} p<0.05,{ }^{* *} p<0.01$ compared to saline treatment by two-way ANOVA followed by Bonferroni post-hoc test. 
A T3 Severe Compression
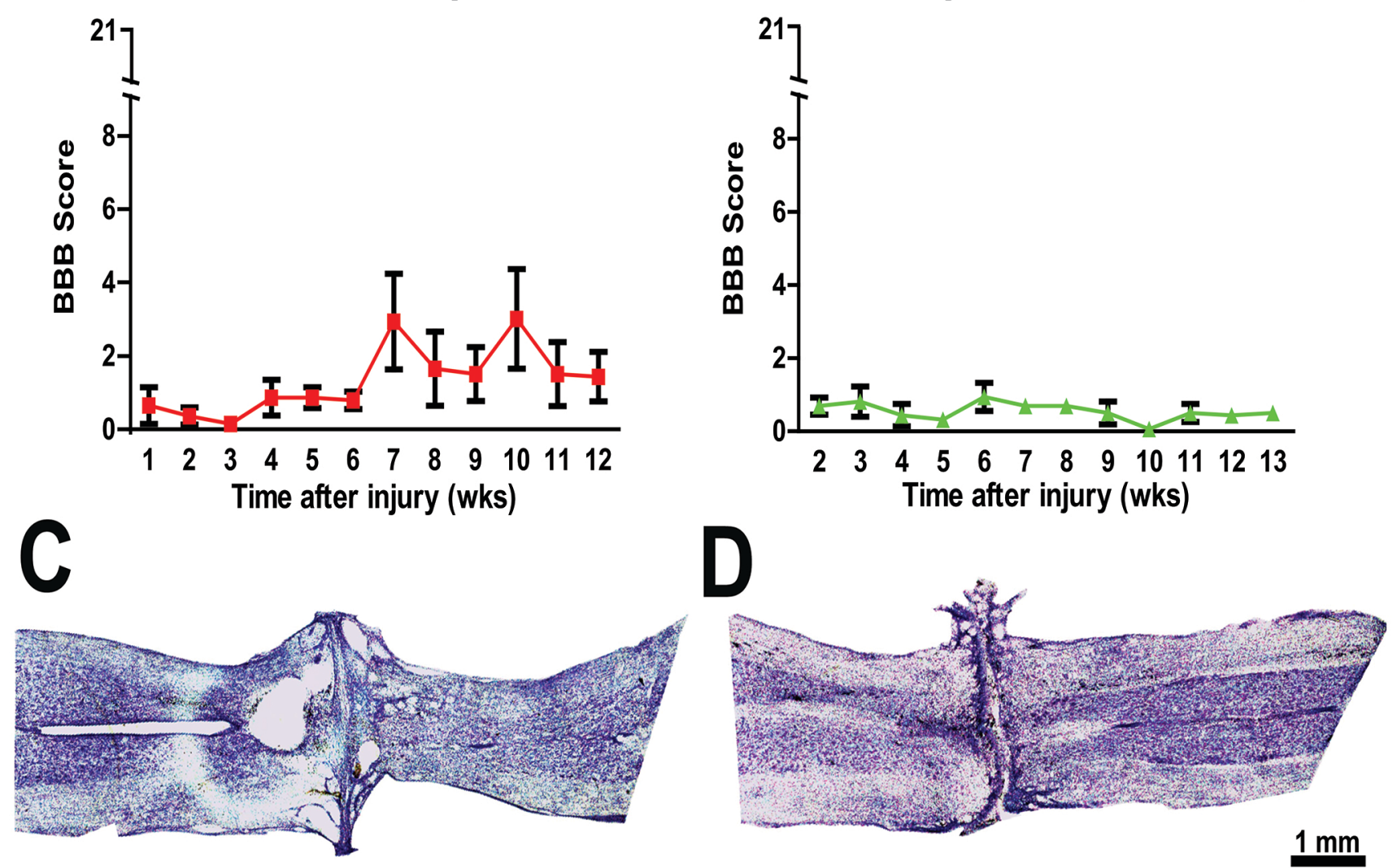

T3 Complete Transection 
A

T3 Compression
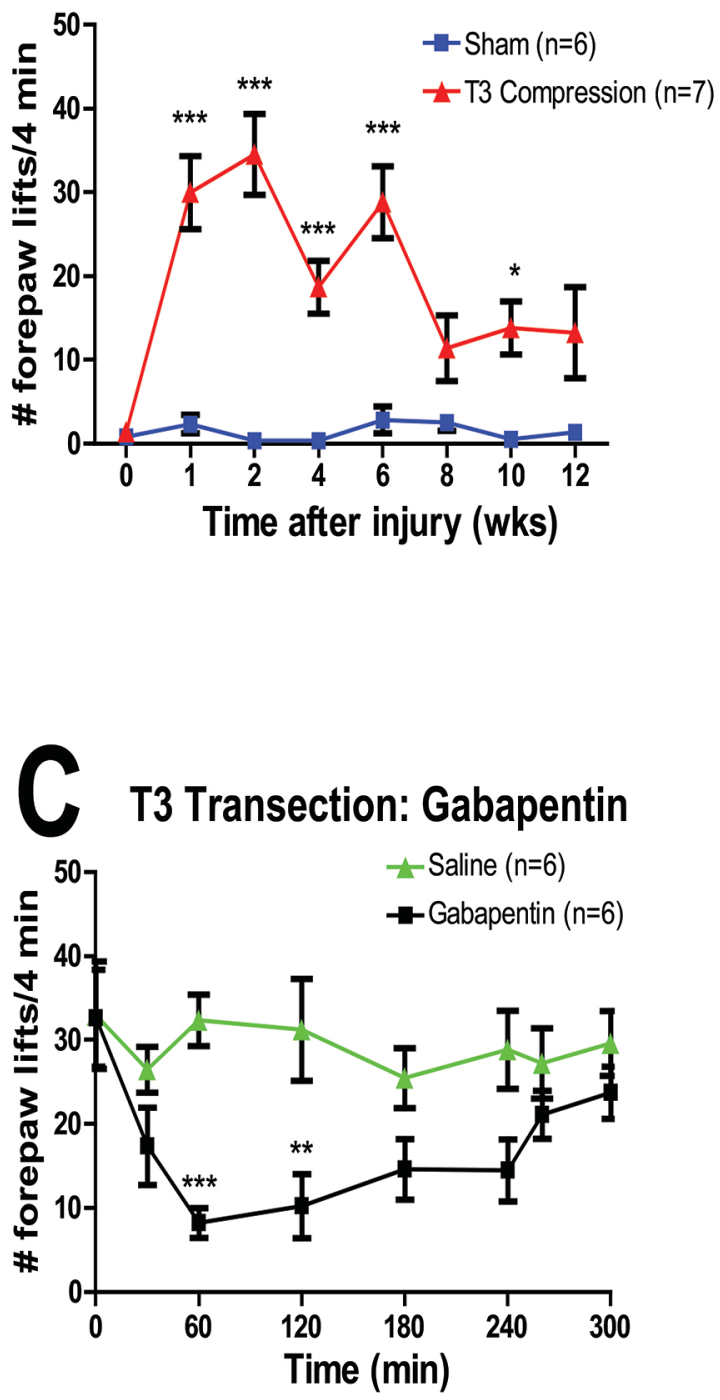

T3 Transection

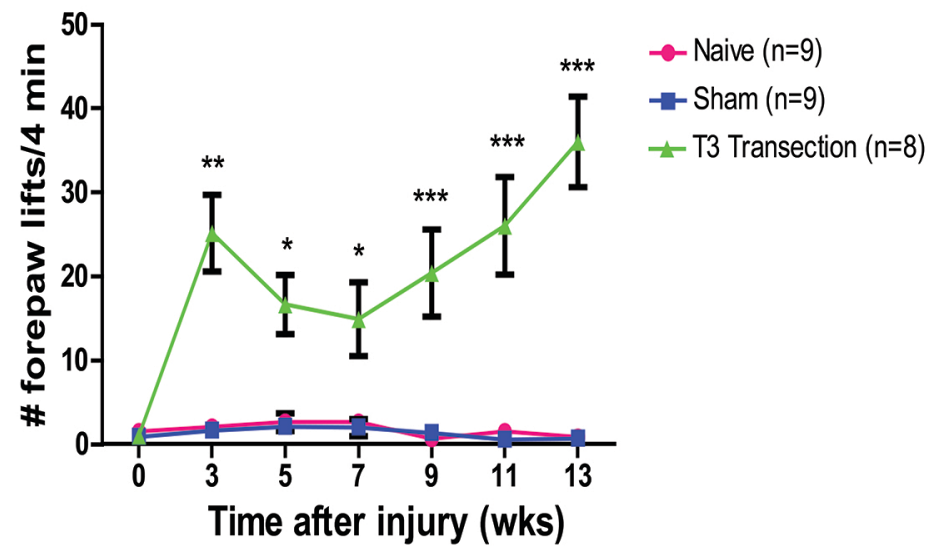

T3 vs. T11 Transection

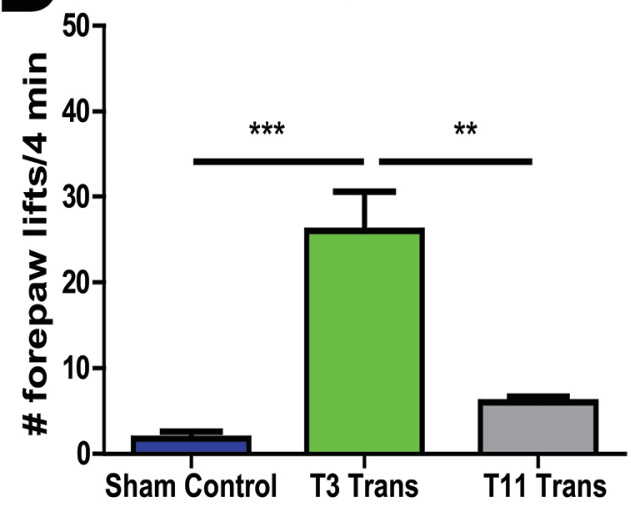

Figure 2 
A

T3 Compression

B

T3 Transection
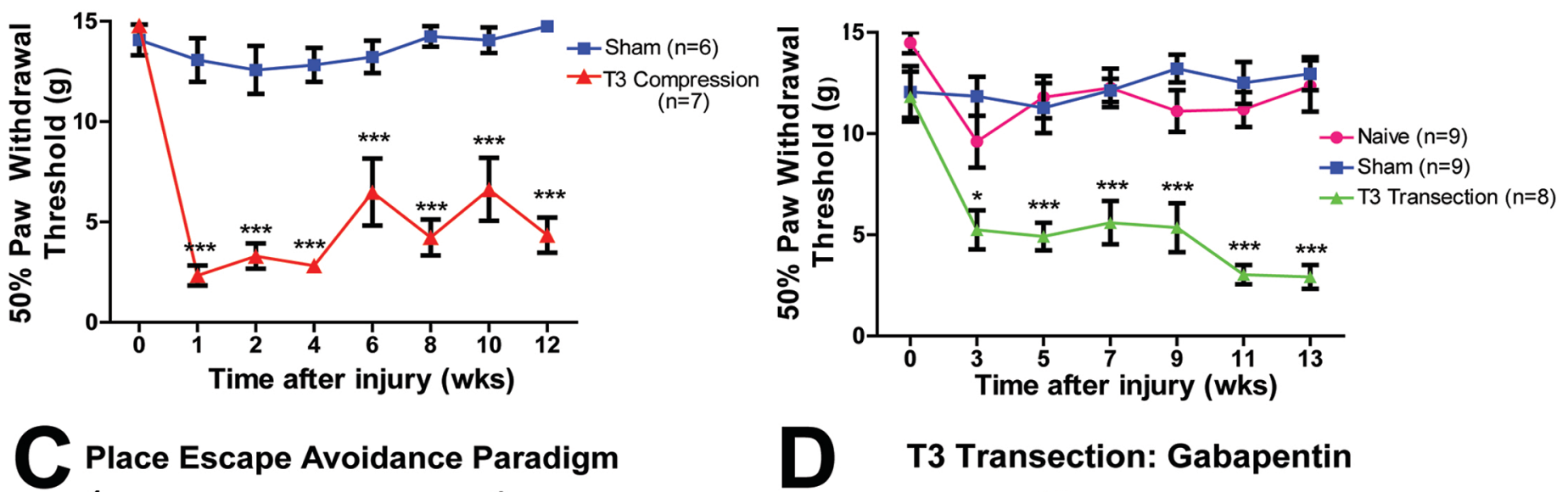

D T3 Transection: Gabapentin
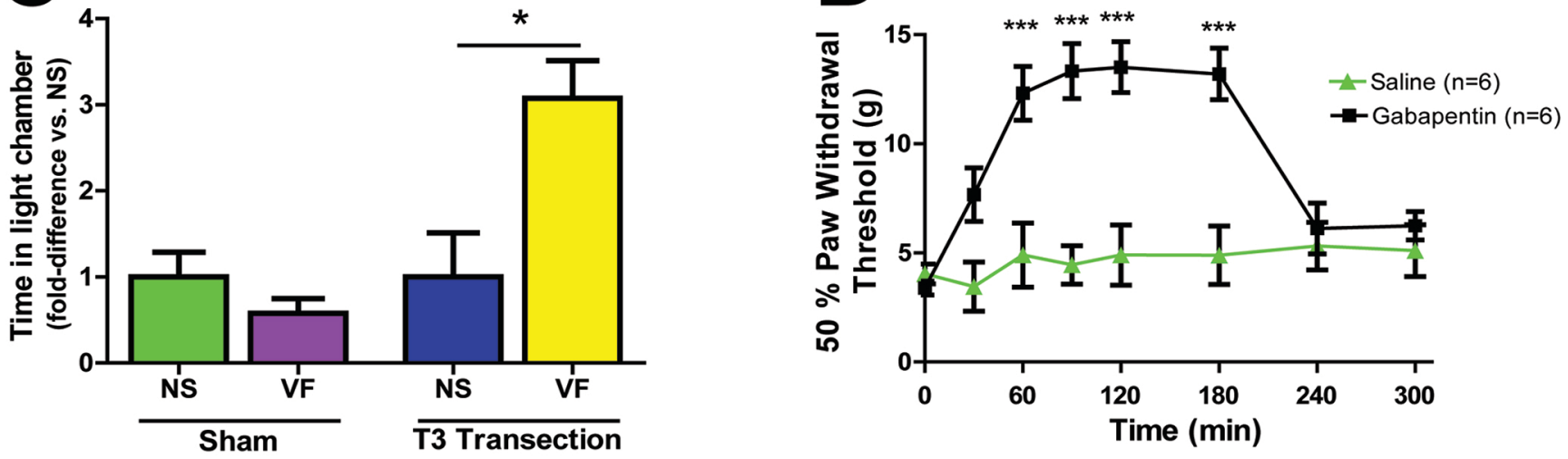

T3 vs. T11 Transection

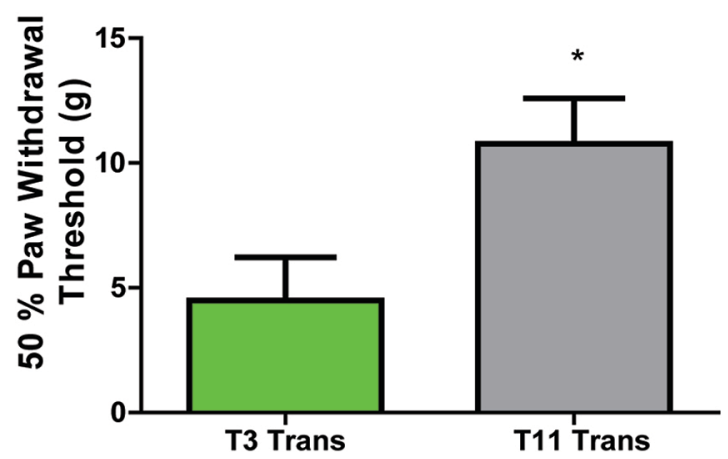




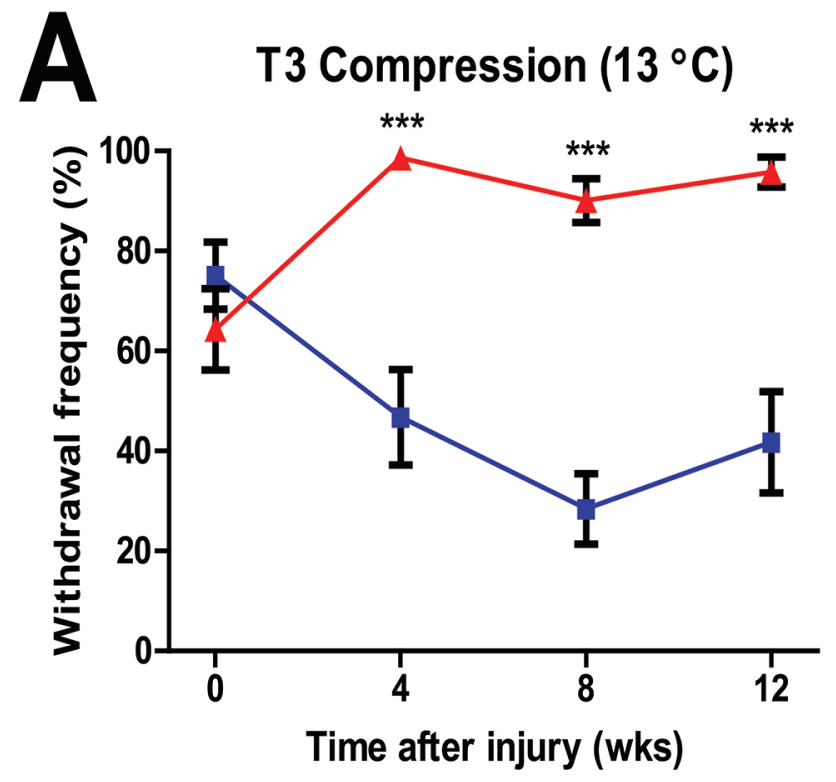

B

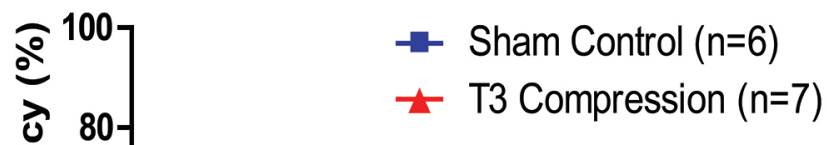

T3 Compression (RT)

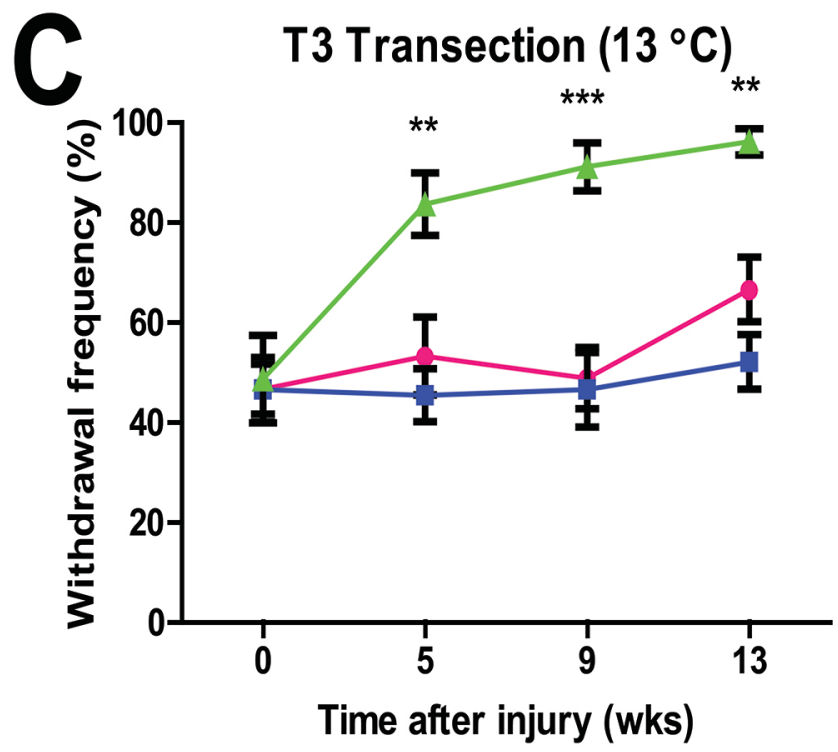

D

T3 Transection (RT)

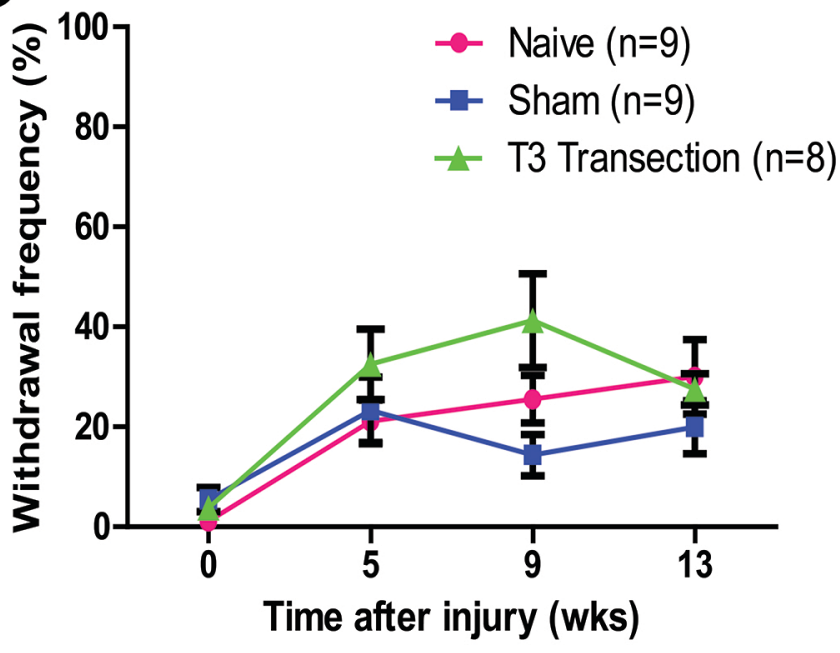

Naive $(n=9)$

Sham Control $(n=9)$

$\square$ T3 Full Transection ( $n=8)$

Figure 4 

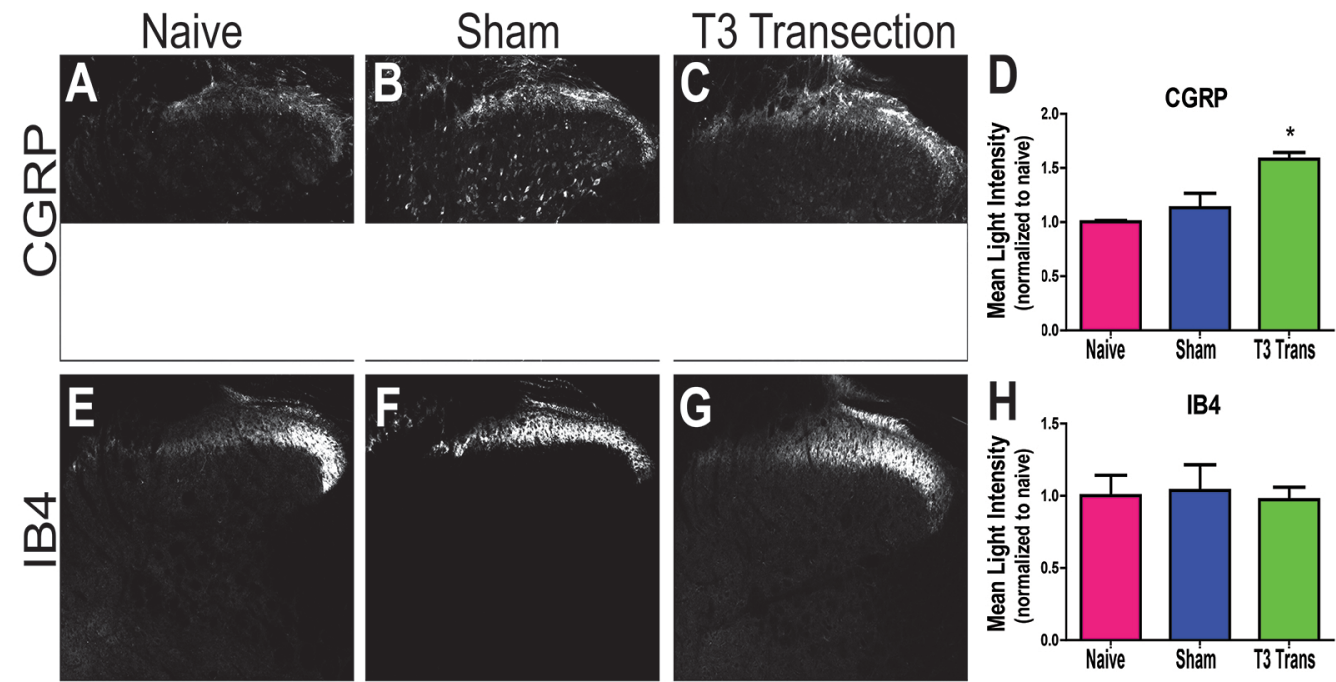

M
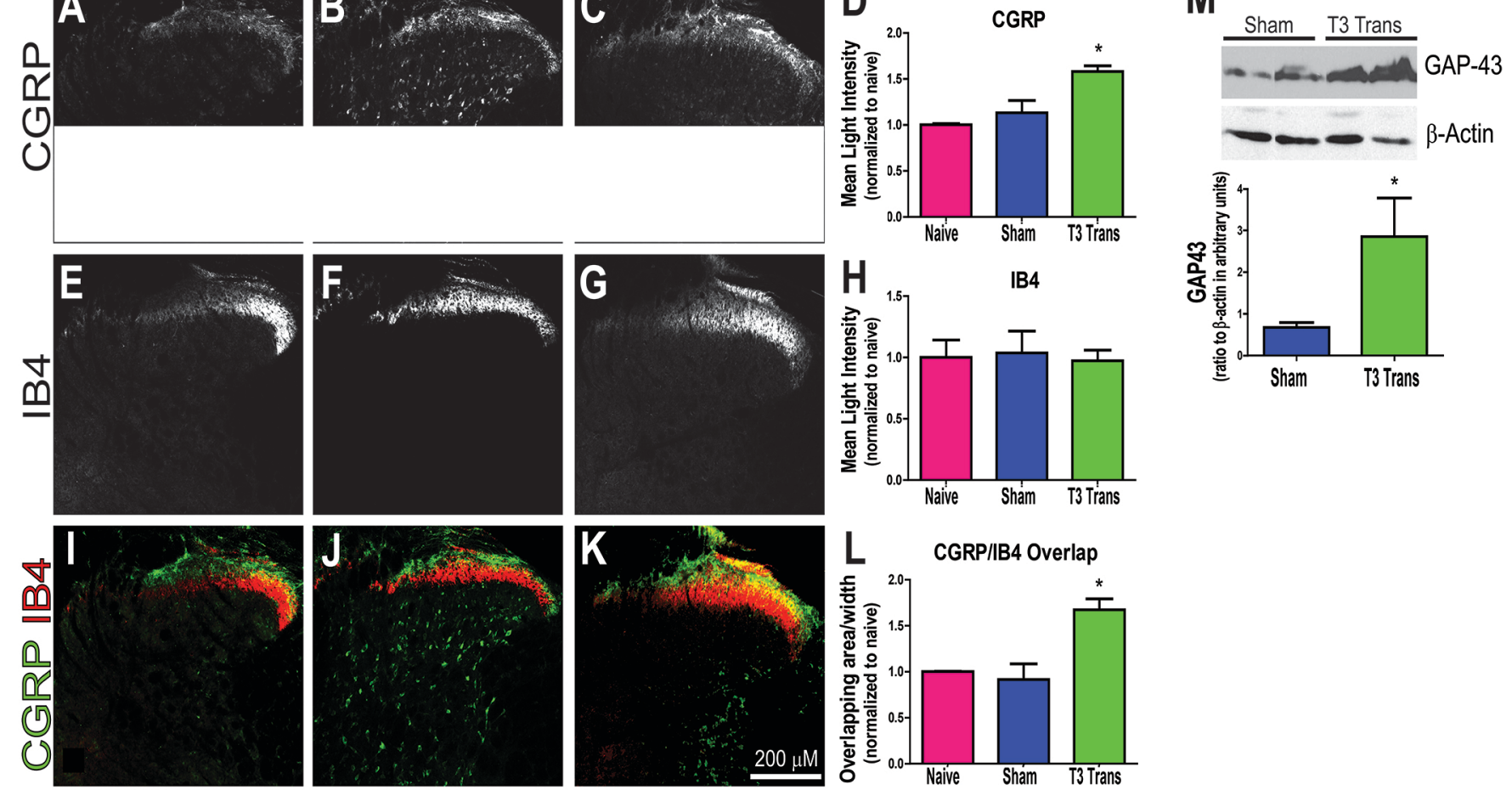

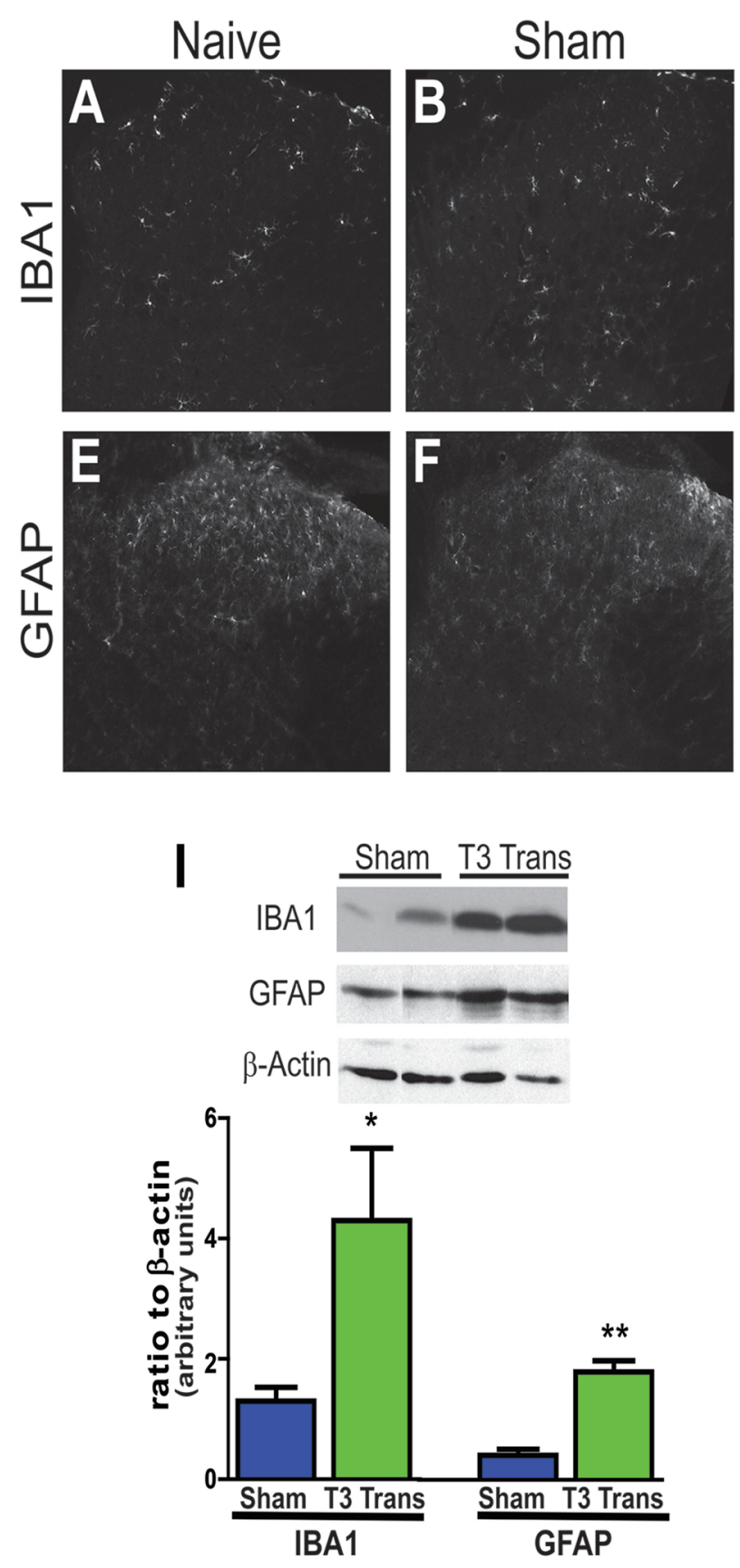

T3 Transection
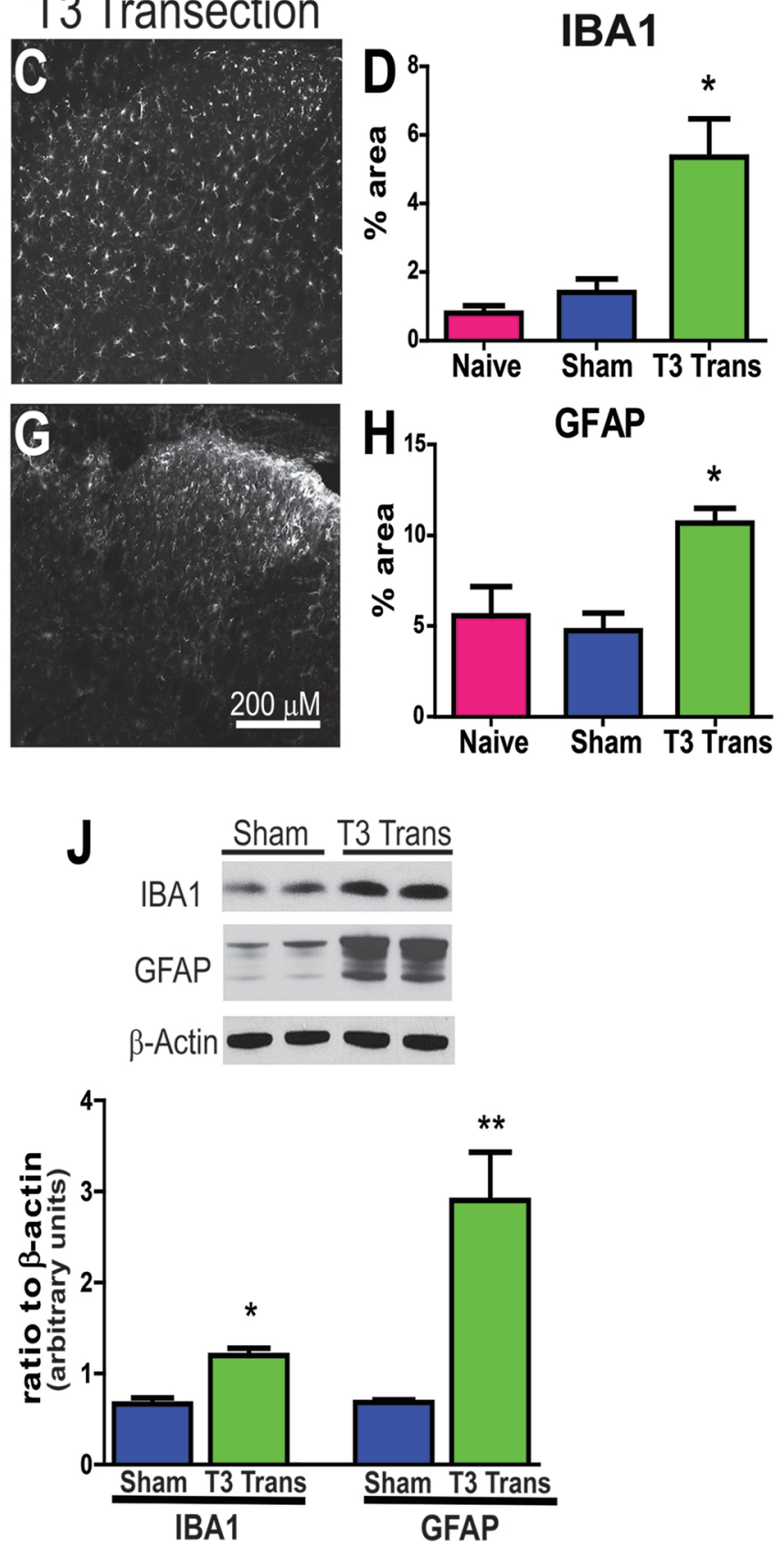
A

Week 4

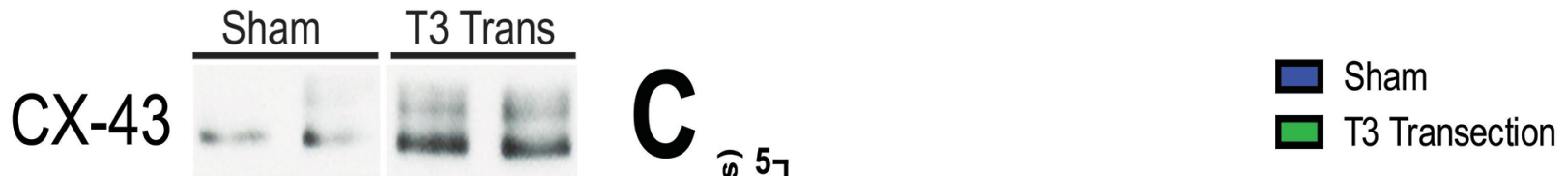

Tuj1

B

Week 8

Sham T3 Trans

CX-43

Tuj1
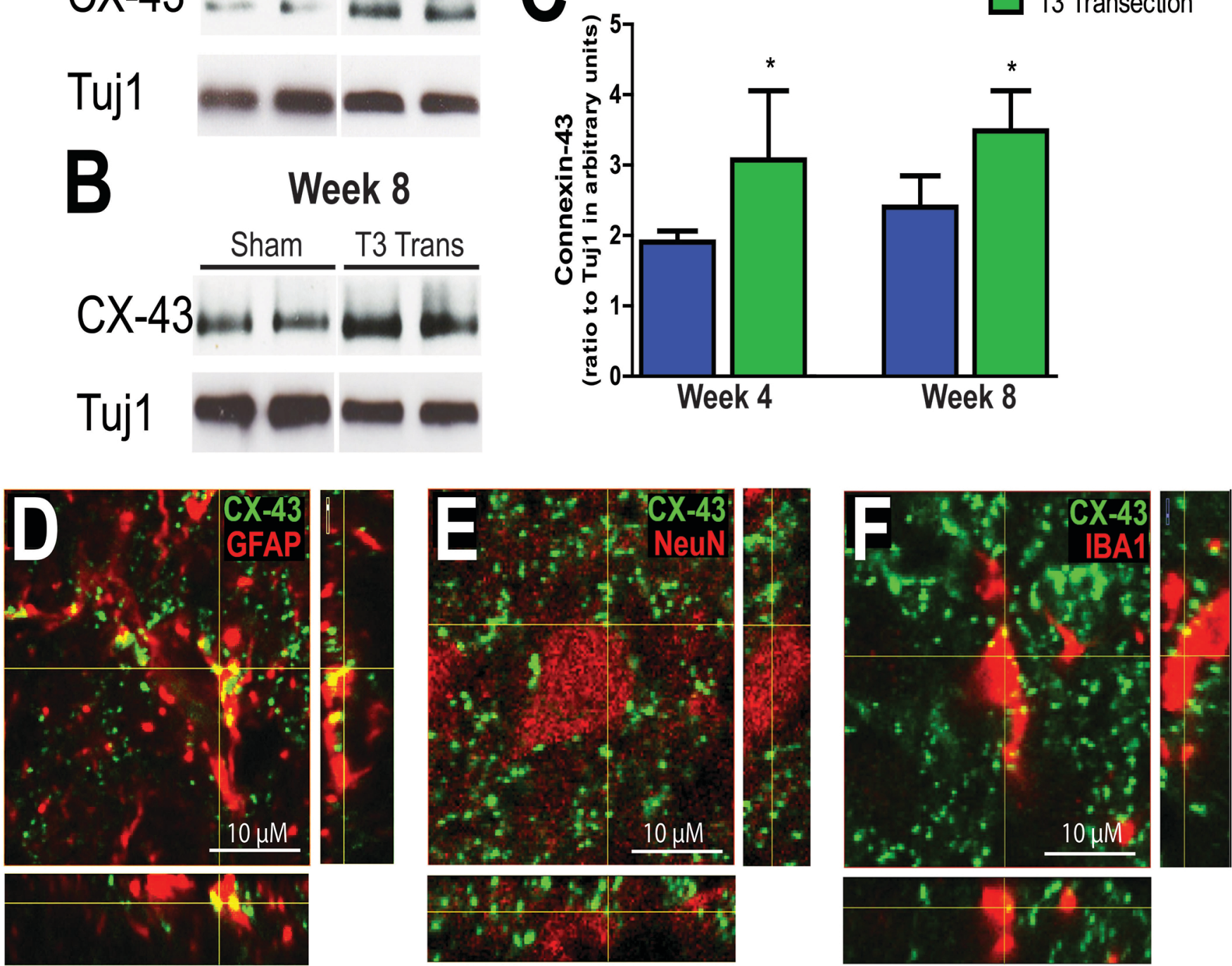

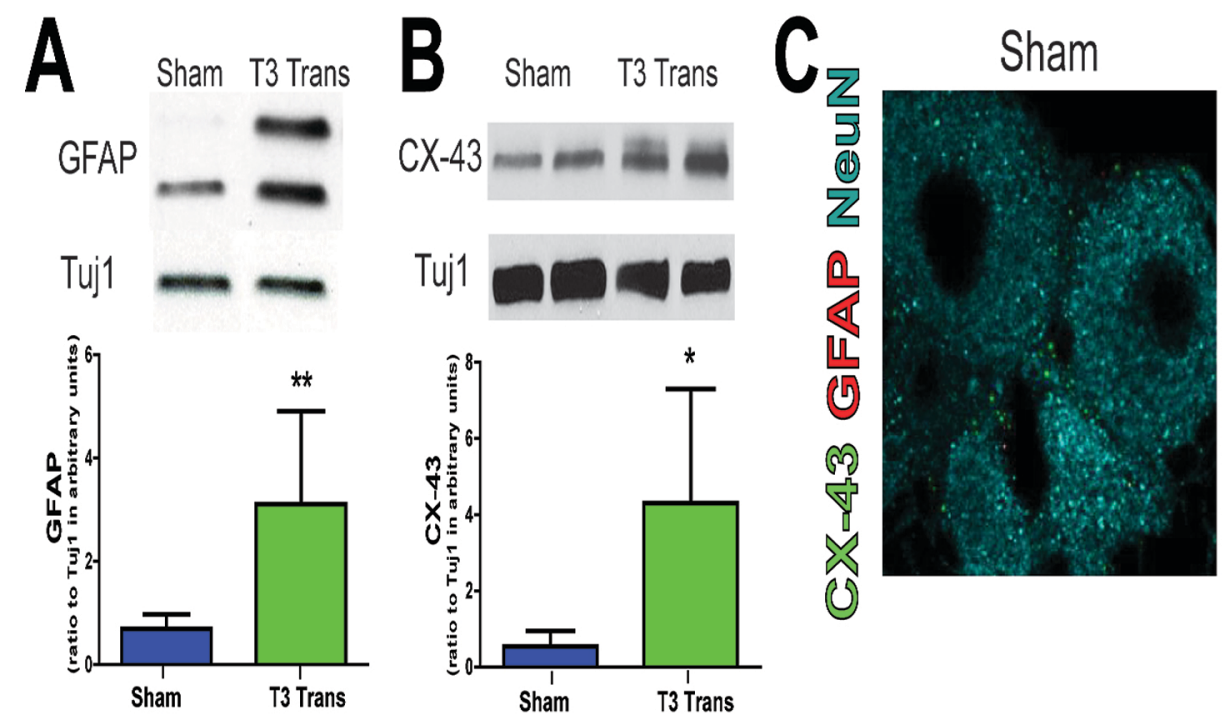

T3 Transection
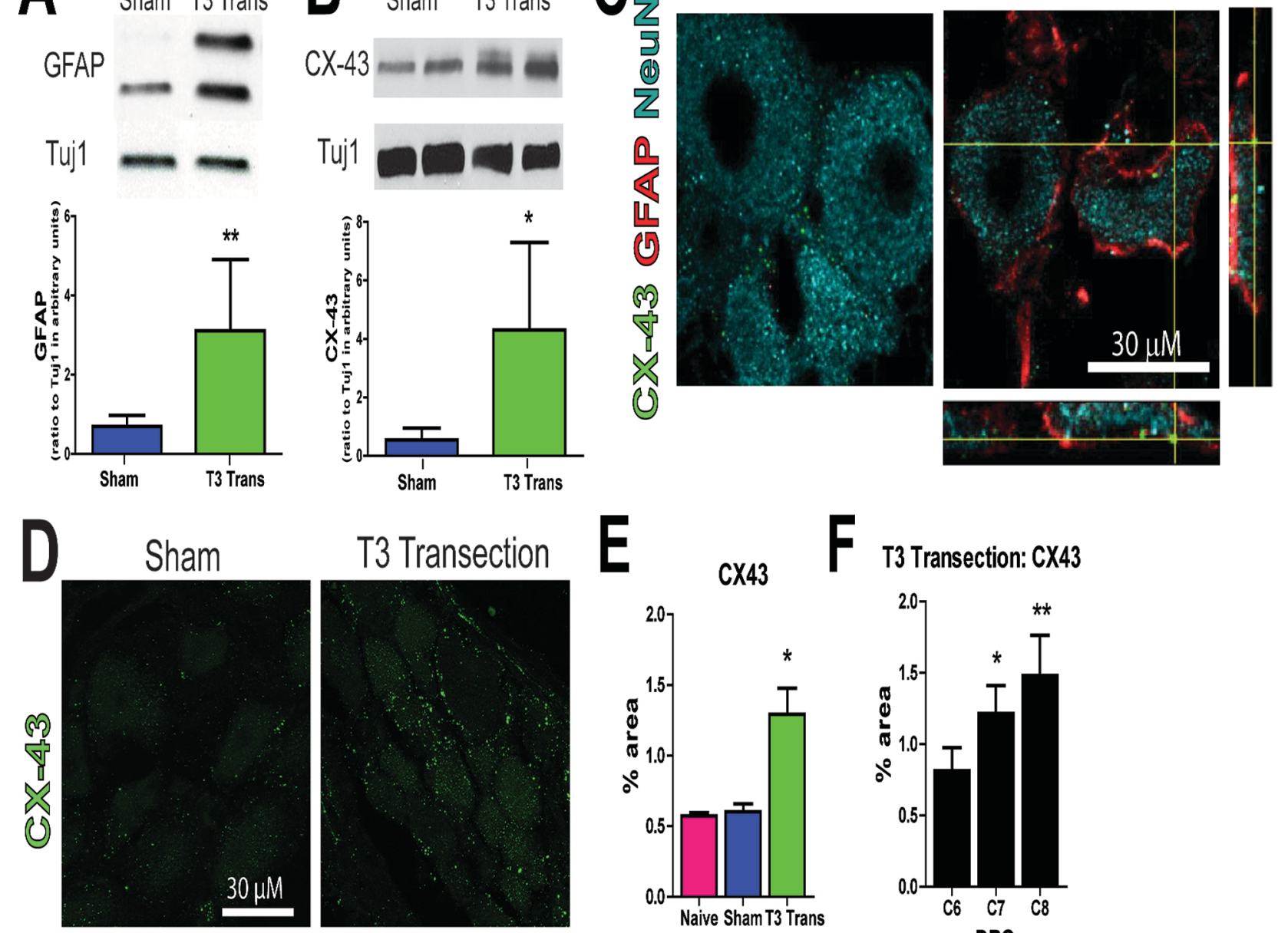

E T3 Transection: CX43

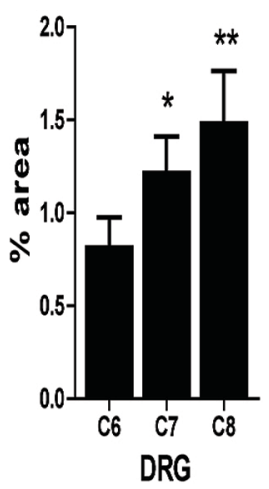



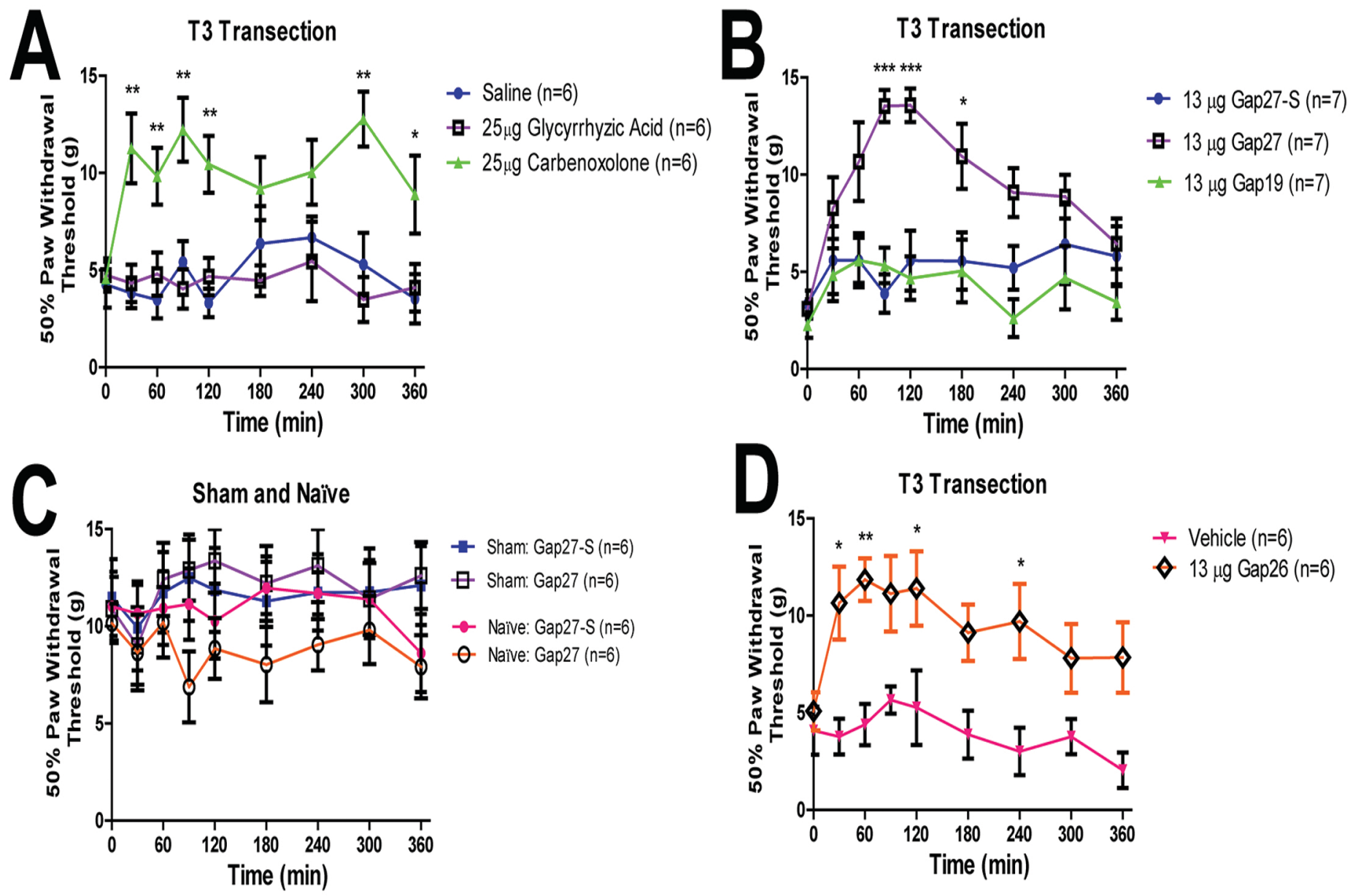